\title{
Antibody resistance of SARS-CoV-2 variants B.1.351 and B.1.1.7
}

https://doi.org/10.1038/s41586-021-03398-2

Received: 25 January 2021

Accepted: 25 February 2021

Published online: 8 March 2021

Check for updates

\author{
Pengfei Wang ${ }^{1,8}$, Manoj S. Nair ${ }^{1,8}$, Lihong Liu ${ }^{1,8}$, Sho Iketani ${ }^{1,2,8}$, Yang Luo', Yicheng Guo', \\ Maple Wang', Jian Yu' ', Baoshan Zhang ${ }^{3}$, Peter D. Kwong ${ }^{3,4}$, Barney S. Graham ${ }^{3}$, \\ John R. Mascola ${ }^{3}$, Jennifer Y. Chang ${ }^{1,5}$, Michael T. Yin ${ }^{1,5}$, Magdalena Sobieszczyk ${ }^{1,5}$, \\ Christos A. Kyratsous ${ }^{6}$, Lawrence Shapiro ${ }^{1,4,7}$, Zizhang Sheng', Yaoxing Huang ${ }^{1 凶}$ \& \\ David D. Ho $\mathbf{H}^{1,2,5 凶}$
}

The COVID-19 pandemic has had widespread effects across the globe, and its causative agent, SARS-CoV-2, continues to spread. Effective interventions need to be developed to end this pandemic. Single and combination therapies with monoclonal antibodies have received emergency use authorization ${ }^{1-3}$, and more treatments are under development $^{4-7}$. Furthermore, multiple vaccine constructs have shown promise ${ }^{8}$, including two that have an approximately $95 \%$ protective efficacy against COVID-19,10. However, these interventions were directed against the initial SARS-CoV-2 virus that emerged in 2019. The recent detection of SARS-CoV-2 variants B.1.1.7 in the UK ${ }^{11}$ and B.1.351 in South Africa ${ }^{12}$ is of concern because of their purported ease of transmission and extensive mutations in the spike protein. Here we show that B.1.1.7 is refractory to neutralization by most monoclonal antibodies against the $\mathrm{N}$-terminal domain of the spike protein and is relatively resistant to a few monoclonal antibodies against the receptor-binding domain. It is not more resistant to plasma from individuals who have recovered from COVID-19 or sera from individuals who have been vaccinated against SARS-CoV-2. The B.1.351 variant is not only refractory to neutralization by most monoclonal antibodies against the $\mathrm{N}$-terminal domain but also by multiple individual monoclonal antibodies against the receptor-binding motif of the receptor-binding domain, which is mostly due to a mutation causing an $\mathrm{E} 484 \mathrm{~K}$ substitution. Moreover, compared to wild-type SARS-CoV-2, B.1.351 is markedly more resistant to neutralization by convalescent plasma (9.4-fold) and sera from individuals who have been vaccinated (10.3-12.4-fold). B.1.351 and emergent variants ${ }^{13,14}$ with similar mutations in the spike protein present new challenges for monoclonal antibody therapies and threaten the protective efficacy of current vaccines.
Considerable evolution of SARS-CoV-2 has occurred since its initial emergence, including the appearance of variants with a D614G mutation ${ }^{15}$ that have become dominant. However, viruses with this mutation alone do not appear to be antigenically distinct ${ }^{16}$. SARS-CoV-2 B.1.1.7-which is also known as 501Y.V1 in the GR clade (Extended Data Fig. 1a) -was first detected in September 2020 in southeast England and rapidly became the dominant variant in the UK, possibly owing to its enhanced transmissibility ${ }^{11}$. This strain has now spread to more than 50 countries and there are indications that it may be more virulent ${ }^{17}$. B.1.1.7 contains eight mutations in the spike gene in addition to the mutation causing the D614G substitution, including mutations that cause two deletions $(\Delta \mathrm{H} 69 / \Delta \mathrm{V} 70$ and $\Delta \mathrm{Y} 144)$ in the $\mathrm{N}$-terminal domain (NTD), one substitution (N501Y) in the receptor-binding domain (RBD) and one substitution $(\mathrm{P} 681 \mathrm{H})$ near the furin cleavage site (Extended Data
Fig. 1b). SARS-CoV-2 B.1.351-which is also known as 501Y.V2 in the GH clade (Extended Data Fig. 1a) - was first detected in late 2020 in Eastern Cape, South Africa ${ }^{12}$. This variant has since become dominant locally, raising the possibility that it too has enhanced transmissibility. B.1.351 contains nine mutations in the spike gene in addition to the mutation causing the D614G substitution, including a cluster of mutations (for example, mutations leading to $\Delta 242-\Delta 244$ and R246I) in the NTD, three substitutions (K417N, E484K and N501Y) in the RBD and one substitution (A701V) near the furin cleavage site (Extended Data Fig. 1b). There is a growing concern that these new variants could impair the efficacy of current monoclonal antibody therapies or vaccines, because many of the mutations are found in the antigenic supersite in the NTD ${ }^{18,19}$ or in the ACE2-binding site (also known as the receptor-binding motif (RBM)) that is a major target of potent virus-neutralizing antibodies.

${ }^{1}$ Aaron Diamond AIDS Research Center, Columbia University Vagelos College of Physicians and Surgeons, New York, NY, USA. ${ }^{2}$ Department of Microbiology and Immunology, Columbia

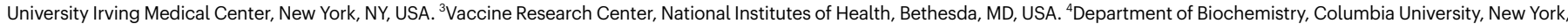

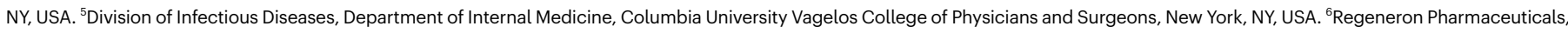
Tarrytown, NY, USA. ${ }^{7}$ Zuckerman Mind Brain Behavior Institute, Columbia University, New York, NY, USA. ${ }^{8}$ These authors contributed equally: Pengfei Wang, Manoj S. Nair, Lihong Liu,

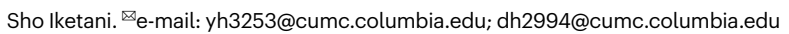




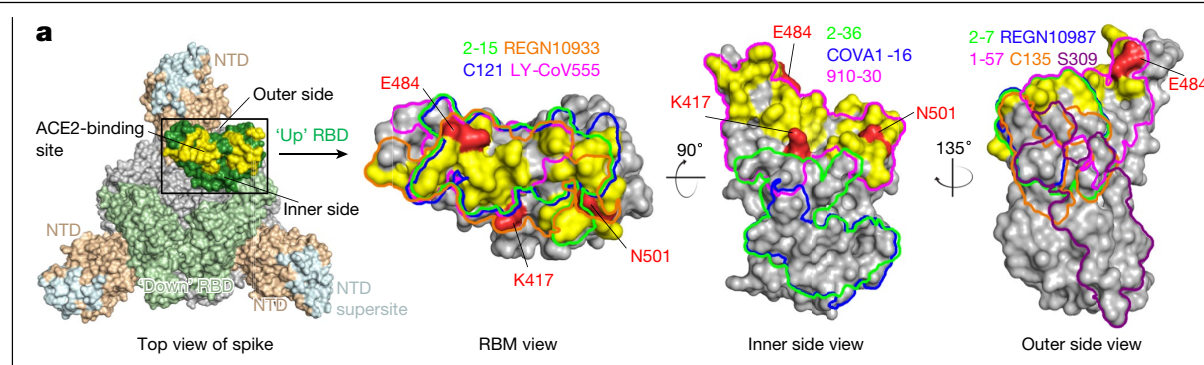

Fig. 1 | Susceptibility of B.1.1.7 and B.1.351 to neutralization by monoclonal antibodies.

a, Footprints of neutralizing monoclonal antibodies on the RBD. Left, top view of SARS-COV-2 spike with one RBD in the 'up' conformation (PDB: 6zgg). The RBD and NTD are coloured green and peach, respectively. The positions of the inner and outer sides are indicated on the up RBD with the ACE2-binding site coloured yellow. Right, the antibody footprints on the RBD. b, Neutralization of B.1.1.7, B.1.351 and wild-type (WA1) viruses by select

b

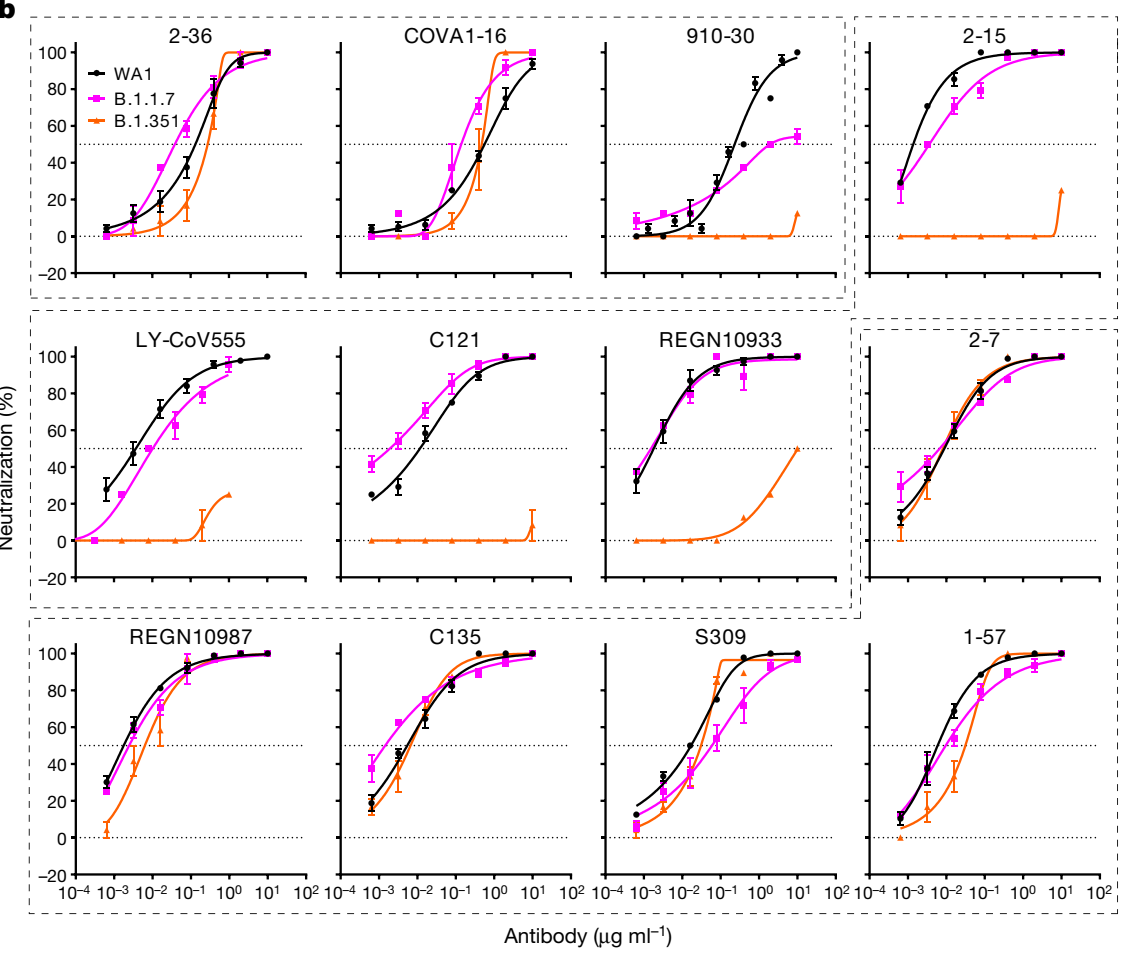
RBD monoclonal antibodies. The horizontal dotted lines on each graph indicate $50 \%$ and $0 \%$ neutralization. The dashed outlines in $\mathbf{b}$ indicate the grouping of the monoclonal antibodies according to their epitopes shown in a. Data are mean \pm s.e.m. of technical triplicates and represent one of two independent experiments. Neutralization by negative control monoclonal antibodies is shown in Extended Data Fig. 2a.

We therefore address this concern by assessing the susceptibility of authentic B.1.1.7 and B.1.351 viruses to neutralization by 30 monoclonal antibodies, plasma from 20 patients convalescing from COVID-19 and sera from 22 individuals who received the Moderna or Pfizer vaccine. In addition, we created vesicular stomatitis virus (VSV)-based SARS-CoV-2 pseudoviruses that contain each of the individual mutations as well as one with all eight mutations of the B.1.1.7 variant (B.1.1.7 $\Delta 8$ ) and another with all nine mutations of the B.1.351 variant (B.1.351 $\Delta$ 9). A total of 18 mutant pseudoviruses were made as previously described ${ }^{20,21}$, and each was found to have a robust titre (Extended Data Fig. 1c) that was adequate for neutralization studies.

\section{Monoclonal antibodies}

We first assayed the neutralizing activity of 12 RBD monoclonal antibodies against the authentic B.1.1.7 and B.1.351 viruses compared with the original SARS-CoV-2 strain in Vero E6 cells, as previously described ${ }^{20,21}$. Three monoclonal antibodies target the 'inner side', four target the RBM and five target the 'outer side' of the RBD. The footprints of these monoclonal antibodies on the RBD are shown in Fig. 1a and their neutralization profiles are shown in Fig. 1b. For neutralization of B.1.1.7, only the activities of $910-30^{22}$ and $\mathrm{S} 309^{5}$ are substantially impaired. For neutralization of B.1.351, however, the activities of 910-30, 2-15 ${ }^{20}$, LY-CoV555 (bamlanivimab) (1,23 $^{1} \mathrm{C1}^{2} 1^{24}$ and REGN10933 (casirivimab) ${ }^{2}$ are completely or markedly abolished. The four monoclonal antibodies that target the RBM are among the most potent SARS-CoV-2-neutralizing antibodies in clinical use or under development. Monoclonal antibodies directed to lower aspects of the inner side $\left(2-36^{20}\right.$ and COVA1-16 $\left.{ }^{25,26}\right)$ or to the 'outer side' retain their activities against B.1.351, including $2-7^{20,27}$, REGN10987 (imdevimab) ${ }^{2}, \mathrm{C}_{135^{24}}$ and S309, which are in clinical use or under development. The results for the neutralization of B.1.1.7 and B.1.351 by these 12 monoclonal antibodies are summarized in Fig. 2a as fold increases or decreases in $50 \%$ inhibitory concentration $\left(\mathrm{IC}_{50}\right)$ relative to the wild-type virus. To understand the specific mutations in the spike gene that are responsible for the observed changes, we also tested the same panel of monoclonal antibodies against pseudoviruses B.1.1.7 $\Delta 8$ and B.1.351 $\Delta$ 9, as well as those containing only single mutations found in B.1.1.7 or B.1.351. The results are displayed in Extended Data Fig. 3 and are summarized in Fig. 2a. There is general agreement in the results for B.1.1.7 and B.1.1.7 $\Delta$, as well as the results for B.1.351 and B.1.351 $\Delta$. The decreased activity of 910-30 against B.1.1.7 is mediated by the N501Y substitution, whereas the slightly impaired activity of S309 remains unexplained. The complete loss of activity of 2-15, LY-CoV555 and $\mathrm{C} 121$ against $\mathrm{B} .1 .351$ is mediated by the $\mathrm{E} 484 \mathrm{~K}$ substitution; the complete loss of activity of 910-30 is mediated by the K417N substitution; and the marked reduction in activity of REGN10933 is mediated by $\mathrm{K} 417 \mathrm{~N}$ and $\mathrm{E} 484 \mathrm{~K}$, as has previously been reported ${ }^{28}$. A structural explanation of how E484K disrupts the binding of 2-15, LY-CoV555 and REGN10933 is presented in Extended Data Fig. 4a.

We also assessed the neutralizing activity of six NTD-directed monoclonal antibodies against B.1.1.7, B.1.351 and wild-type viruses. Both B.1.1.7 and B.1.351 are markedly resistant to neutralization by antibodies 5-24 and $4-8^{20}$, as well as by $4 \mathrm{~A}^{29}$, all of which target the antigenic supersite in the $\mathrm{NTD}^{18}$ (Fig. 2b). The activities of 2-17, 4-19 and 5-7 ${ }^{20}$ 
a

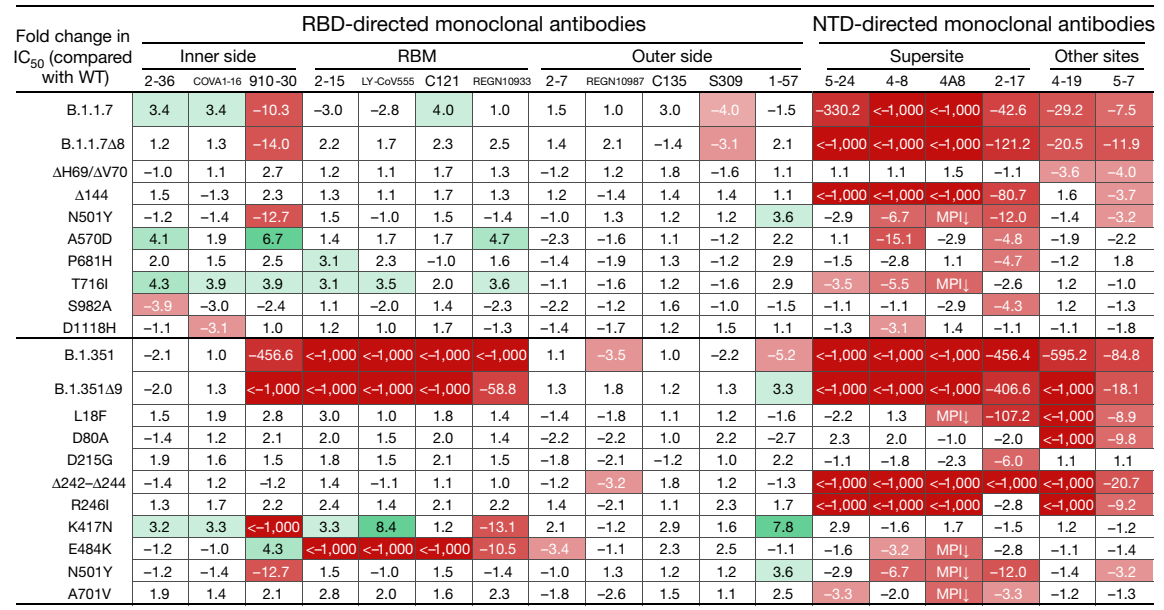

b

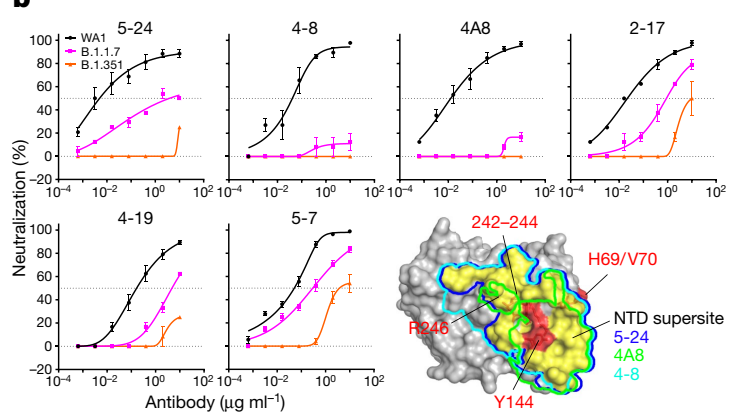

c

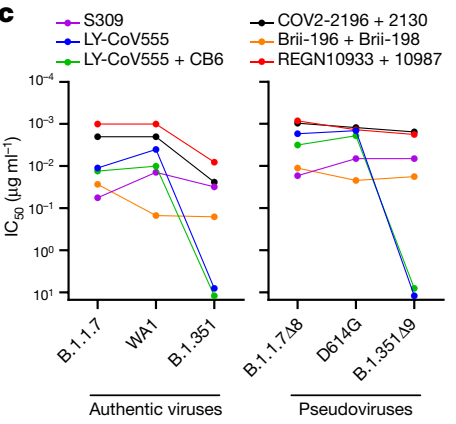

Fig. 2 | Susceptibility of B.1.1.7 and B.1.351 to neutralization by monoclonal antibodies (continued). a, Fold increase or decrease in $\mathrm{IC}_{50}$ of neutralizing monoclonal antibodies against B.1.1.7, B.1.351, B.1.1.7 $\Delta$, B.1.351 $\Delta$ 9 and single-mutation pseudoviruses relative to the wild-type (WT) virus, presented as a heat map in which darker colours indicate a greater change. MPI with a downward arrow indicates that the maximum percentage inhibition is substantially reduced, confounding $\mathrm{IC}_{50}$ calculations. Red, resistance $>3$-fold; green, sensitization $>3$-fold. $\mathbf{b}$, Neutralization of B.1.1.7, B.1.351 and wild-type viruses by NTD-directed monoclonal antibodies, the footprints of which are delineated by the colour tracings in the inset. The horizontal dotted lines on each graph indicate $50 \%$ and $0 \%$ neutralization. Data are mean \pm s.e.m. of technical triplicates and represent one of two independent experiments. $c$, Changes in neutralization $\mathrm{IC}_{50}$ of authorized or investigational therapeutic monoclonal antibodies against B.1.1.7, B.1.351 and wild-type (WA1) viruses (left) as well as B.1.1.7 $\Delta 8$, B.1.351 $\Delta 9$ and wild-type (D614G) pseudoviruses (right). are variably impaired, particularly against B.1.351. To understand the specific mutations that are responsible for the observed changes, we then tested these monoclonal antibodies against pseudoviruses containing only single mutations found in B.1.1.7 or B.1.351 (Extended Data Fig. 3). The results are summarized in Fig. 2a as a fold increase or decrease relative to the wild-type virus. We found that the resistance of B.1.1.7 to most NTD-directed monoclonal antibodies is largely conferred by $\Delta \mathrm{Y} 144$, whereas the resistance of B.1.351 is largely conferred by $\Delta 242-\Delta 244$ and/or R246I. Amino acid residues $144,242-244$ and 246 all fall within the NTD supersite ${ }^{18,19}$ (Fig. 2b; details are shown in Extended Data Fig. 4b).

We next tested the neutralizing activity of 12 additional RBD monoclonal antibodies, including ones from our own collection (1-20, 4-20, 2-4, 2-43, 2-30 and 2-38) ${ }^{20}$ as well as CB6 (etesevimab) ${ }^{3,6}$, COV2-2196 and COV2-2130 ${ }^{7}$, Brii-196 and Brii-198 ${ }^{4}$, and REGN10985. The results against B.1.1.7, B.1.351 and wild-type viruses are shown in Extended Data Fig. 5a, and the detailed findings against the single-mutation pseudoviruses are shown in Extended Data Fig. 3. The fold changes in neutralization $\mathrm{IC}_{50}$ titres relative to the wild-type virus are tabulated in Extended Data Fig. 5b. Here, we discuss only the results for monoclonal antibodies in clinical development. The activity of $\mathrm{CB} 6$ is rendered inactive against B.1.351 because of the K417N substitution. Brii-196 and COV2-2130 are essentially unaffected by the new variants; the activities of Brii-198 and COV2-2196 are diminished by 14.6 -fold and 6.3-fold, respectively, against B.1.351 but not against B.1.1.7.

We then examined, in a single experiment, the neutralizing activity of monoclonal antibody therapies in clinical use or under clinical investigation against B.1.1.7, B.1.351 and wild-type viruses, as well as against B.1.1.7 $\Delta 8$, B.1.351 $\Delta 9$ and wild-type pseudoviruses. The results for the single monoclonal antibodies LY-CoV555 and S309, as well as for combination regimens of REGN10933 and REGN10987, LY-CoV555 and CB6, Brii-196 and Brii-198, and COV2-2196 and COV2-2130, are shown in
Extended Data Fig. 6 and summarized in Fig. 2c. LY-CoV555, alone or in combination with CB6, is no longer able to neutralize B.1.351. Although REGN10933 and REGN10987 and COV2-2196 and COV2-2130 are seemingly unaffected against variant pseudoviruses, there are noticeable decreases in their activity against the B.1.351 authentic virus. Although S309 and the combination of Brii-196 and Brii-198 are not significantly impaired, their potencies are noticeably lower (Fig. 2c). These findings suggest that antibody treatment of this virus might need to be modified in localities in which B.1.351 and related variants ${ }^{13,14}$ are prevalent, and highlight the importance of combination antibody therapy to address the expanding antigenic diversity of SARS-CoV-2.

\section{Convalescent plasma}

We obtained plasma from 20 patients convalescing from COVID-19 more than one month after documented SARS-CoV-2 infection in the spring of 2020. Each plasma sample was then assayed for neutralization against B.1.1.7, B.1.351 and wild-type viruses. Figure 3a shows that most (16 out of 20 ) plasma samples lost more than 2.5 -fold neutralizing activity against B.1.351, while maintaining activity against B.1.1.7. Only plasma from P7, P10, P18 and P20 retain neutralizing activities similar to those against the wild-type virus. These results are summarized as fold increase or decrease in $50 \%$ inhibitory dilution $\left(\mathrm{ID}_{50}\right)$ in Fig. $3 \mathrm{~b}$. Furthermore, the magnitude of the drop in plasma neutralization is better seen in Fig. 3c, showing no loss of activity against B.1.1.7 but substantial loss against B.1.351 (9.4-fold).

Every plasma sample was also tested against each mutant pseudovirus, and those findings are shown in Extended Data Fig. 7 and summarized in Fig. 3b, c. Eight samples show a decrease of more than 2.5 -fold in neutralizing activity against B.1.1.7 $\Delta 8$, in contrast to the results for neutralization of B.1.1.7. These discrepant results highlight a previous observation ${ }^{20}$ that pseudovirus neutralization does not always faithfully 


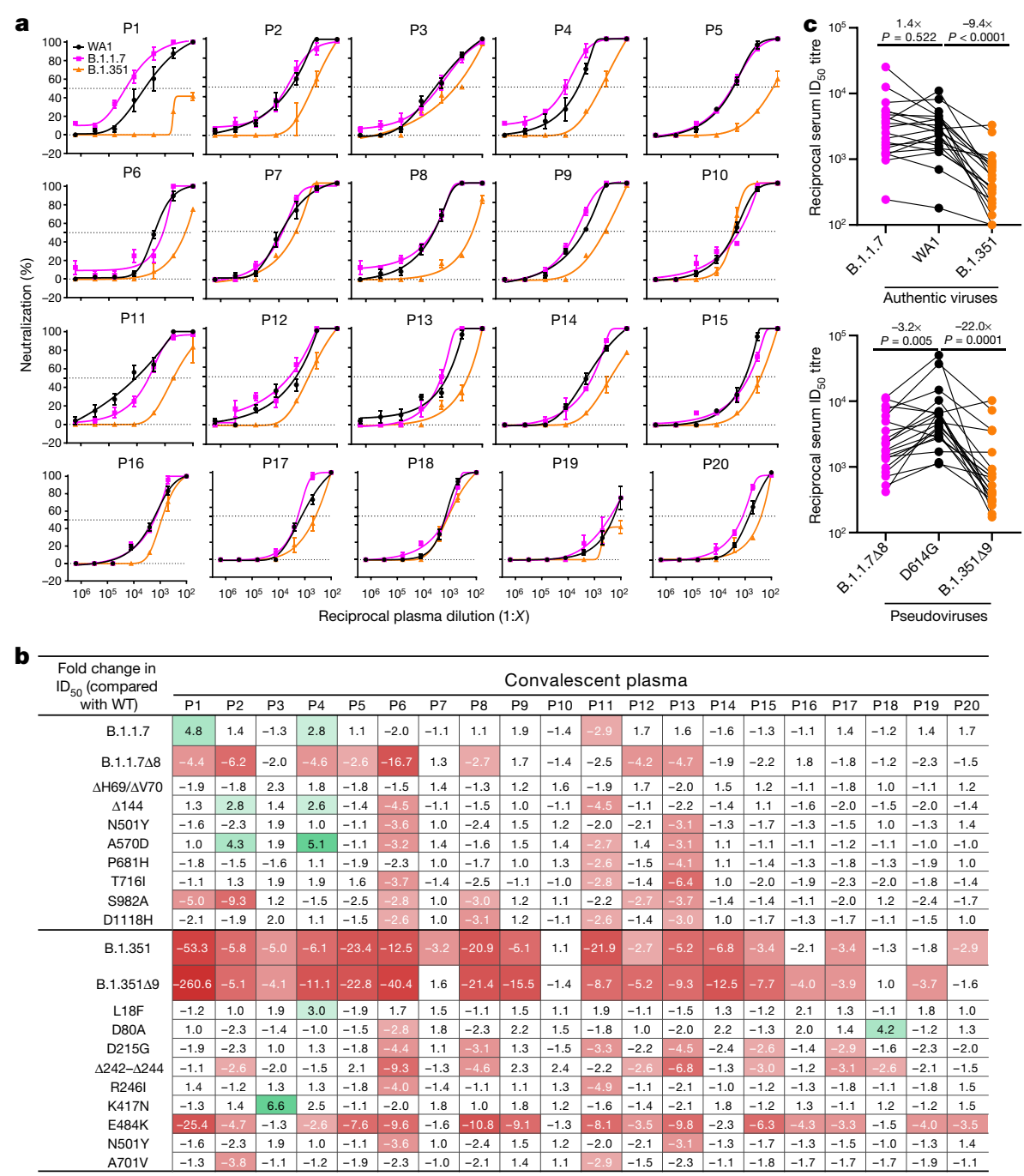

Fig. 3 | B.1.351 is more resistant to neutralization by convalescent plasma from patients.

a, Neutralization results for 20 convalescent plasma samples (P1-P20) against B.1.1.7, B.1.351 and wild-type viruses. The horizontal dotted lines on each graph indicate $50 \%$ and $0 \%$ neutralization. Reciprocal plasma dilutions are given as $1: X$, in which $X$ is the value on the $x$ axis. Data are mean \pm s.e.m. of technical triplicates. Neutralization by healthy donor plasma is shown in Extended Data Fig. $2 b$. b, Fold increase or decrease in neutralization $\mathrm{ID}_{50}$ of B.1.1.7 and B.1.351 viruses, B.1.1.7 $\Delta 8$, B.1.351 $\Delta 9$ and single-mutation pseudoviruses relative to the wild-type virus presented as a heat map in which darker colours indicate a greater change. Red, resistance $>2.5$-fold; green, sensitization $>2.5$-fold. c, Change in reciprocal plasma neutralization $\mathrm{ID}_{50}$ values of convalescent plasma against B.1.1.7, B.1.351, B.1.1.7 8 and B.1.351 $\Delta 9$ relative to the wild-type virus. Mean fold changes in $\mathrm{ID}_{50}$ values relative to the wild-type virus are written above the $P$ values. Statistical analysis was performed using a Wilcoxon matched-pairs signed-rank test. Two-tailed $P$ values are reported.

recapitulate live virus neutralization. The loss of plasma neutralizing activity against B.1.351 could be largely attributed to the E484K substitution (Fig. 3b), which has been shown to attenuate the neutralizing activity of convalescent sera ${ }^{30}$. Our findings here suggests that this mutation in the RBM is situated in an immunodominant epitope for most individuals infected with SARS-CoV-2. It is also interesting to note that sera such as P7, P10 and P18 contain neutralizing antibodies that are essentially unperturbed by the multitude of spike mutations found in these two new variants (Fig. 3b). A detailed analysis of the antibody repertoire of these samples against the viral spike could be informative.

\section{Sera from vaccinated individuals}

Sera were obtained from 12 participants of a phase-I clinical trial of the Moderna SARS-Co-2 mRNA-1273 vaccine ${ }^{9}$ conducted at the NIH. These volunteers received two immunizations with the vaccine $(100 \mu \mathrm{g})$ on days 0 and 28 , and blood was collected on day 43 . Additional sera from vaccinated individuals were obtained from 10 individuals who received the Pfizer BNT162b2 COVID-19 vaccine ${ }^{10}$ under emergency use authorization at the clinical dose on days 0 and 21. Blood was collected on day 28 or later.

Each serum sample from vaccinated individuals was assayed for neutralization against B.1.1.7, B.1.351 and wild-type viruses. Figure 4a shows no loss of neutralizing activity against B.1.1.7, whereas every sample lost activity against B.1.351. These results are quantified and tabulated as the fold increase or decrease in neutralization $\mathrm{ID}_{50}$ titres in
Fig. $4 \mathrm{~b}$, and the extent of the decrease in neutralization activity is more evident in Fig. 4c. Overall, the neutralizing activity against B.1.1.7 was essentially unchanged, but significantly lower against B.1.351 (12.4-fold for the Moderna vaccine; 10.3 -fold for the Pfizer vaccine).

Every serum from vaccinated indivdiuals was also tested against each mutant pseudovirus, and the results are presented in Extended Data Fig. 8 and summarized in Fig. 4b, c. No single mutation in B.1.1.7 had an appreciable effect on the neutralizing activity of sera from vaccinated individuals. The loss of neutralizing activity against B.1.351 $\Delta 9$ is largely consistent with the loss in neutralization of the B.1.351 live virus. A major contributor to the resistance to neutralization of this variant virus appears to be the E484K substitution (Fig. 4b), indicating that this mutation in the RBM is situated in an immunodominant epitope recognized by all sera from the vaccinated individuals studied.

\section{Discussion}

Both SARS-CoV-2 variants B.1.1.7 and B.1.351 are raising concerns not only because of their increase transmissibility but also because of their extensive mutations in the spike gene that could lead to antigenic changes that are detrimental to monoclonal antibody therapies and protection afforded by vaccines. It is of equal concern that another variant known as P.1 or 501Y.V3 is increasing rapidly in Brazil and spreading far beyond ${ }^{13,14}$. P.1 contains three substitutions (K417T, E484K and N501Y) at the same RBD residues as B.1.351. Much of our findings on B.1.351 would probably be similar for this emergent variant. N501Y is 


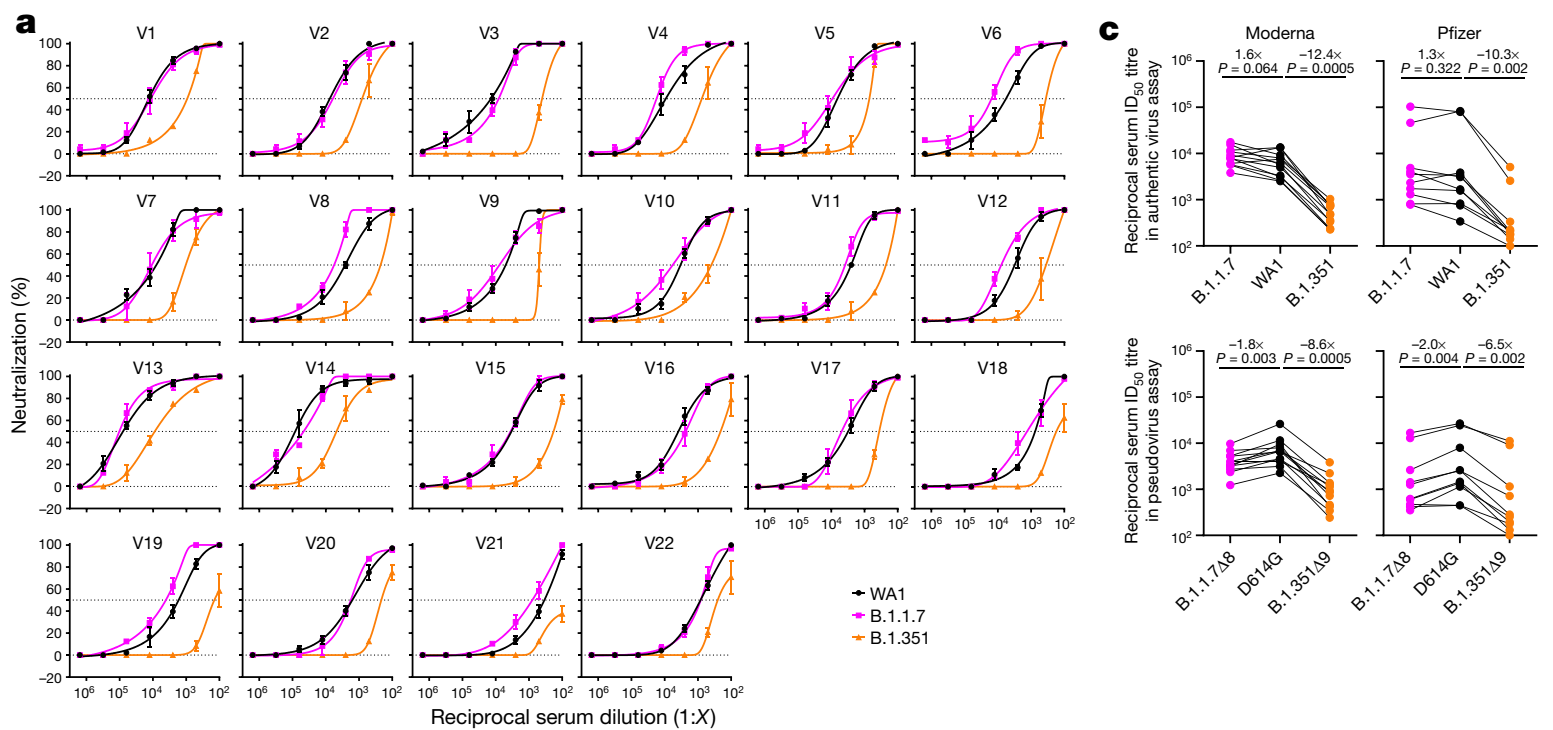

\begin{tabular}{|c|c|c|c|c|c|c|c|c|c|c|c|c|c|c|c|c|c|c|c|c|c|c|}
\hline \multirow{2}{*}{$\begin{array}{l}\text { Fold change in } \\
\mathrm{ID}_{50} \text { (compared } \\
\text { with WT) }\end{array}$} & \multicolumn{12}{|c|}{ Moderna } & \multicolumn{10}{|c|}{ Pfizer } \\
\hline & $\mathrm{V} 1$ & V2 & V3 & V4 & V5 & V6 & V7 & V8 & V9 & V10 & V11 & V12 & V13 & V14 & V15 & V16 & V17 & V18 & V19 & V20 & V21 & V22 \\
\hline B.1.1.7 & -1.1 & -1.2 & -1.7 & 1.9 & 1.5 & 2.7 & 1.4 & 2.1 & 1.5 & 1.7 & 1.5 & 2.5 & 1.3 & -1.8 & 1.1 & -1.7 & 1.6 & 1.7 & 2.4 & 1.1 & 2.3 & -1.0 \\
\hline B.1.1.7 $\Delta 8$ & -2.7 & -2.2 & -3.0 & -1.2 & -1.7 & -1.9 & -1.2 & -1.9 & -1.4 & 1.0 & -1.2 & -1.8 & -1.6 & -1.9 & -2.1 & -1.8 & 1.1 & -2.4 & -2.3 & -3.2 & -1.1 & 1.1 \\
\hline$\Delta \mathrm{H} 69 / \Delta \mathrm{V} 70$ & 1.4 & 1.4 & -1.3 & 1.2 & -1.3 & -1.1 & 1.9 & 1.1 & 1.5 & -1.4 & 1.3 & 1.3 & -1.4 & 1.3 & -1.0 & -1.0 & -1.5 & -1.3 & 1.3 & 1.3 & -1.4 & 2.1 \\
\hline$\Delta 144$ & -1.1 & -1.2 & -1.4 & 2.1 & -1.2 & -1.2 & -1.1 & -1.2 & -1.2 & -1.1 & 1.1 & -1.3 & -1.2 & -1.7 & -1.2 & -1.2 & -1.3 & 1.1 & -1.2 & -1.6 & 1.1 & -1.3 \\
\hline N501Y & 1.5 & 1.1 & -1.8 & 1.6 & -2.0 & 1.9 & 2.2 & -2.0 & -1.2 & 4.6 & 2.9 & -1.2 & -1.2 & 1.2 & -2.1 & -1.6 & -1.6 & -1.5 & -1.1 & -2.4 & -1.4 & 1.0 \\
\hline A570D & 1.4 & 2.2 & 1.2 & 2.4 & 1.7 & 1.6 & 2.2 & 1.5 & 1.0 & 1.5 & 1.4 & 1.6 & 1.2 & 1.5 & 1.5 & 2.6 & 1.2 & 1.3 & 1.8 & 1.1 & -1.2 & 1.4 \\
\hline $\mathrm{P} 681 \mathrm{H}$ & 2.2 & 1.2 & -1.7 & -1.5 & -1.5 & 1.0 & 1.1 & -1.4 & 1.0 & -1.1 & 1.1 & 1.1 & 1.2 & 1.2 & -1.3 & 1.1 & -1.1 & -1.1 & -1.0 & -2.1 & -1.3 & -1.0 \\
\hline T716I & 1.1 & -1.1 & -1.1 & 1.6 & 1.3 & 1.3 & 1.8 & 1.1 & 1.0 & 1.6 & 1.2 & 1.4 & 1.7 & 1.4 & 1.3 & 1.1 & 1.1 & 1.3 & 1.1 & 1.6 & 1.1 & 1.1 \\
\hline S982A & -2.3 & -1.5 & -2.6 & -1.8 & -2.0 & -1.6 & -1.3 & -2.5 & -1.7 & -1.5 & -1.5 & -1.5 & -1.2 & -1.6 & -1.6 & -1.7 & -1.5 & -1.9 & -1.6 & -2.4 & -2.0 & -1.3 \\
\hline $\mathrm{D} 1118 \mathrm{H}$ & -1.2 & 1.1 & -1.2 & -1.4 & 1.1 & 1.0 & 1.0 & -1.5 & -1.2 & -1.1 & 1.0 & 1.1 & -1.2 & -1.2 & -1.6 & -1.6 & -1.2 & -1.5 & -1.2 & -1.3 & -1.7 & -1.3 \\
\hline B.1.351 & -14.4 & $\mid-9.4$ & -28.8 & -12.1 & $\mid-8.7$ & -17.0 & $|-7.7|$ & -11.6 & -10.2 & -8.4 & $|-11.1|$ & -8.8 & -9.0 & -17.5 & -18.4 & -18.5 & -9.3 & -4.3 & -11.9 & -7.4 & $<-3.4$ & -3.7 \\
\hline B. $1.351 \Delta 9$ & -6.9 & -8.4 & -22.7 & -11.0 & -6.1 & -7.7 & -2.9 & -10.0 & -4.8 & -3.2 & $|-13.0|$ & -6.6 & -3.0 & -2.2 & -9.2 & -3.5 & -2.5 & -7.5 & -10.4 & -4.5 & $<-4.5$ & -2.5 \\
\hline L18F & 1.9 & 1.0 & -1.8 & -1.1 & -1.3 & 1.0 & 3.3 & -1.5 & 1.0 & 1.2 & 1.8 & 1.0 & 1.2 & -1.3 & 1.2 & 1.2 & 1.0 & -1.4 & 1.4 & -1.4 & -1.5 & 1.4 \\
\hline D80A & 1.2 & 1.5 & -1.1 & 2.1 & 1.1 & 1.5 & 1.8 & -1.5 & 1.4 & 3.0 & 1.3 & 1.1 & 1.8 & 1.0 & 1.2 & 1.4 & -1.5 & -1.8 & 1.0 & -1.8 & -1.3 & 1.1 \\
\hline D215G & -1.3 & 1.1 & 1.1 & -1.2 & 1.3 & 1.2 & -1.1 & -2.9 & -1.1 & 2.7 & 1.1 & -1.3 & -1.1 & 1.1 & -1.8 & -2.0 & -1.2 & -1.8 & -1.3 & 1.0 & -1.2 & 1.1 \\
\hline$\Delta 242-\Delta 244$ & -3.6 & 1.0 & -1.3 & -1.8 & -1.7 & -1.3 & -1.7 & -1.7 & -1.4 & -1.5 & -1.6 & -1.6 & -1.4 & -1.9 & -1.4 & -1.3 & 1.5 & 1.1 & -1.3 & -2.6 & -1.6 & -1.8 \\
\hline R246I & -1.6 & 1.1 & -2.0 & -1.1 & -1.7 & -1.2 & 1.1 & -2.1 & $\mid-1.3$ & -1.1 & 1.0 & -1.7 & -1.5 & -1.2 & 1.6 & 1.0 & 1.3 & 2.0 & 2.9 & -4.0 & 1.0 & -1.1 \\
\hline $\mathrm{K} 417 \mathrm{~N}$ & 1.6 & 1.4 & -1.1 & 1.0 & 1.0 & -1.2 & 1.7 & -1.3 & 1.1 & 1.4 & -1.3 & 1.5 & 1.4 & 1.8 & 1.2 & 1.4 & -1.5 & 1.9 & 2.0 & 1.0 & -1.8 & 1.6 \\
\hline E484K & -3.0 & -2.3 & -3.9 & -4.0 & -1.4 & -2.8 & -1.3 & -3.3 & -2.2 & -2.6 & -3.2 & -1.8 & -1.9 & -2.7 & -2.1 & -1.6 & -2.9 & -11.3 & -3.3 & -3.2 & -3.1 & -1.8 \\
\hline N501Y & 1.5 & 1.1 & -1.8 & 1.6 & -2.0 & 1.9 & 2.2 & -2.0 & -1.2 & 4.6 & 2.9 & -1.2 & -1.2 & 1.2 & -2.1 & -1.6 & -1.6 & -1.5 & -1.1 & -2.4 & -1.4 & 1.0 \\
\hline A701V & -1.1 & -1.2 & -1.9 & -2.2 & -1.7 & -1.6 & -1.4 & -1.7 & 1.2 & 1.1 & 2.1 & -1.1 & -1.2 & -1.3 & -1.2 & -1.1 & -2.1 & -1.5 & \begin{tabular}{|l|}
-1.4 \\
\end{tabular} & 1.1 & -2.2 & -1.5 \\
\hline
\end{tabular}

Fig. 4 | B.1.351 is more resistant to neutralization by sera from individuals vaccinated with the Moderna or Pfizer vaccine. a, Neutralization profiles for 22 serum samples obtained from individuals who received the SARS-CoV-2 vaccine made by Moderna (V1-V12) or Pfizer (V13-V22) against B.1.1.7, B.1.351 and wild-type viruses. The horizontal dotted lines on each graph indicate $50 \%$ and $0 \%$ neutralization. Reciprocal serum dilutions are given as $1: X$, in which $X$ is the value on the $x$ axis. Data are mean \pm s.e.m. of technical triplicates and represent one of two independent experiments. b. Fold change in serum neutralization $\mathrm{ID}_{50}$ of B.1.1.7 and B.1.351 viruses, B.1.1.7 $\Delta 8$, B.1.351 $\Delta 9$ and single-mutation pseudoviruses relative to the wild-type virus, presented as a heat map in which darker colours indicate greater change. Red, resistance $>2.5$-fold; green, sensitization $>2.5$-fold. $c$, Change in reciprocal serum ID $_{50}$ values for individuals who received the Moderna and $P$ fizer vaccines against B.1.1.7, B.1.351, B.1.1.7 $\Delta 8$ and B.1.351 $\Delta 9$ relative to the wild-type virus. Mean fold change in $\mathrm{ID}_{50}$ relative to the wild-type virus is written above the $P$ values. Statistical analysis was performed using a Wilcoxon matched-pairs signed-rank test. Two-tailed $P$ values are reported.

neutralization activity (Fig. 2c). Several other monoclonal antibodies in development are similarly impaired (Fig. 2a, c and Extended Data Fig. 5b) against this variant. Decisions on the use of these monoclonal antibodies will depend heavily on the local prevalence of B.1.351 or variants with an $\mathrm{E} 484 \mathrm{~K}$ substitution, thus highlighting the importance of viral genomic surveillance worldwide and proactive development of next-generation antibody therapeutics, including combinations that target antigenically distinct epitopes.

Convalescent plasma from patients infected with SARS-CoV-2 from early in the pandemic show no significant change in neutralizing activity against B.1.1.7, but the reduction against B.1.351 is remarkable (Fig. 3b, c). This relative resistance is mostly due to the $\mathrm{E} 484 \mathrm{~K}$ substitution, which is shared by the B.1.351 and P.1 variants ${ }^{12-14}$. Again, in areas in which such viruses are common, one would have a concern about re-infection, as other studies are also suggesting ${ }^{35,36}$. This apprehension is heightened 
by the recent observation from the Novavax vaccine trial in South Africa that recipients who received the placebo with previous SARS-CoV-2 infection were not protected against a subsequent exposure to B.1.351 ${ }^{37,38}$. Even more disturbing is the situation in Manaus, Brazil, where a second wave of infection due to P.1 is sweeping through a population that was already $76 \%$ seropositive owing to prior infection in the spring of $2020^{39}$.

As for the ramifications of our findings for the protective efficacy of current SARS-CoV-2 vaccines, the neutralizing activity of sera from vaccinated individuals against B.1.1.7 is largely intact and no adverse effects on current vaccines are expected (Fig. 4c), consistent with conclusions that were reached by other studies ${ }^{34,40,41}$. On the other hand, the loss in activity by 10.3-12.4-fold against B.1.351 is larger than results that were reported using mutant pseudoviruses ${ }^{34,42,43}$. Taken together, the overall findings are troubling, particularly in light of recent reports that both the Novavax and Johnson \& Johnson vaccines showed a substantial drop in efficacy in South Africa ${ }^{37,38}$.

The recent emergence of B.1.1.7, B.1.351 and P.1 marks the beginning of SARS-CoV-2 antigenic drift. This conclusion is supported by the data presented here, illustrating how so many of these changes in the spike protein conferred resistance to antibody neutralization, and by studies reporting similar mutations in the spike gene selected by antibody pressure in vivo ${ }^{44-46}$. Mutationally, this virus is going in a direction that could ultimately lead to escape from our current therapeutic and prophylactic interventions that are directed against the viral spike protein. If the rampant spread of the virus continues and more critical mutations accumulate, then we may be condemned to chasing after the evolving SARS-CoV-2 continually, as we have long done for influenza virus. Such considerations require that we stop virus transmission as quickly as is feasible, by redoubling our mitigation measures and by expediting vaccine rollout.

\section{Online content}

Any methods, additional references, Nature Research reporting summaries, source data, extended data, supplementary information, acknowledgements, peer review information; details of author contributions and competing interests; and statements of data and code availability are available at https://doi.org/10.1038/s41586-021-03398-2.

1. Chen, P. et al. SARS-CoV-2 neutralizing antibody LY-CoV555 in outpatients with COVID-19. N. Engl. J. Med. 384, 229-237 (2021).

2. Hansen, J. et al. Studies in humanized mice and convalescent humans yield a SARS-CoV-2 antibody cocktail. Science 369, 1010-1014 (2020).

3. Gottlieb, R. L. et al. Effect of bamlanivimab as monotherapy or in combination with etesevimab on viral load in patients with mild to moderate COVID-19: a randomized clinical trial. J. Am. Med. Assoc. 325, 632-644 (2021).

4. Ju, B. et al. Human neutralizing antibodies elicited by SARS-CoV-2 infection. Nature $\mathbf{5 8 4}$ $115-119$ (2020).

5. Pinto, D. et al. Cross-neutralization of SARS-CoV-2 by a human monoclonal SARS-CoV antibody. Nature 583, 290-295 (2020).

6. Shi, R. et al. A human neutralizing antibody targets the receptor-binding site of SARS-CoV-2. Nature 584, 120-124 (2020).

7. Zost, S. J. et al. Potently neutralizing and protective human antibodies against SARS-CoV-2. Nature 584, 443-449 (2020)

8. Krammer, F. SARS-CoV-2 vaccines in development. Nature 586, 516-527 (2020).

9. Anderson, E. J. et al. Safety and immunogenicity of SARS-CoV-2 mRNA-1273 vaccine in older adults. N. Engl. J. Med. 383, 2427-2438 (2020).

10. Polack, F. P. et al. Safety and efficacy of the BNT162b2 mRNA COVID-19 vaccine. N. Engl. J. Med. 383, 2603-2615 (2020).

11. Rambaut, A. et al. Preliminary genomic characterisation of an emergent SARS-CoV-2 lineage in the UK defined by a novel set of spike mutations. https://virological.org/t/ preliminarygenomic-characterisation-of-an-emergent-sars-cov-

2-lineage-in-the-uk-defined-by-a-novel-set-ofspike-mutations/563 (2020).

12. Tegally, H. et al. Emergence and rapid spread of a new severe acute respiratory syndrome-related coronavirus 2 (SARS-CoV-2) lineage with multiple spike mutations in South Africa. Preprint at https://doi.org/10.1101/2020.12.21.20248640 (2020).

13. Faria, N. R. et al. Genomic characterisation of an emergent SARS-CoV-2 lineage in Manaus: preliminary findings. Preprint at https://virological.org/t/genomic-characterisationof-an-emergent-sars-cov-2-lineage-in-manaus-preliminary-findings/586 (2021).
14. Naveca, F. et al. Phylogenetic relationship of SARS-CoV-2 sequences from Amazonas with emerging Brazilian variants harboring mutations $\mathrm{E} 484 \mathrm{~K}$ and $\mathrm{N} 501 \mathrm{Y}$ in the spike protein. Preprint at https://virological.org/t/phylogenetic-relationship-of-sars-cov2-sequences-from-amazonas-with-emerging-brazilian-variants-harboring-mutations-e48 4k-and-n501y-in-the-spike-protein/585 (2021).

15. Korber, B. et al. Tracking changes in SARS-CoV-2 spike: evidence that D614G increases infectivity of the COVID-19 virus. Cell 182, 812-827 (2020).

16. Hou, Y. J. et al. SARS-CoV-2 D614G variant exhibits efficient replication ex vivo and transmission in vivo. Science 370, 1464-1468 (2020).

17. Davies, N. G. et al. Increased hazard of death in community-tested cases of SARS-CoV-2 variant of concern 202012/01. Preprint at https://doi.org/10.1101/2021.02.01.21250959 (2021).

18. Cerutti, G. et al. Potent SARS-CoV-2 neutralizing antibodies directed against spike $\mathrm{N}$-terminal domain target a single supersite. Cell Host Microbe (in the press).

19. McCallum, M. et al. N-terminal domain antigenic mapping reveals a site of vulnerability for SARS-CoV-2. Preprint at https://doi.org/10.1101/2021.01.14.426475 (2021).

20. Liu, L. et al. Potent neutralizing antibodies against multiple epitopes on SARS-CoV-2 spike. Nature 584, 450-456 (2020).

21. Wang, P. et al. SARS-CoV-2 neutralizing antibody responses are more robust in patients with severe disease. Emerg. Microbes Infect. 9, 2091-2093 (2020).

22. Banach, B. B. et al. Paired heavy and light chain signatures contribute to potent SARS-CoV-2 neutralization in public antibody responses. Preprint at https://doi. org/10.1101/2020.12.31.424987 (2021).

23. Jones, B. E. et al. LY-CoV555, a rapidly isolated potent neutralizing antibody, provides protection in a non-human primate model of SARS-CoV-2 infection. Preprint at https:// doi.org/10.1101/2020.09.30.318972 (2020)

24. Robbiani, D. F. et al. Convergent antibody responses to SARS-CoV-2 in convalescent individuals. Nature 584, 437-442 (2020).

25. Brouwer, P. J. M. et al. Potent neutralizing antibodies from COVID-19 patients define multiple targets of vulnerability. Science 369, 643-650 (2020).

26. Liu, H. et al. Cross-neutralization of a SARS-CoV-2 antibody to a functionally conserved site is mediated by avidity. Immunity 53, 1272-1280 (2020).

27. Cerutti, G. et al. Structural basis for accommodation of emerging B.1.351 and B.1.1.7 variants by two potent SARS-CoV-2 neutralizing antibodies. Preprint at https://doi.org/ 10.1101/2021.02.21.432168 (2021).

28. Starr, T. N. et al. Prospective mapping of viral mutations that escape antibodies used to treat COVID-19. Science 371, 850-854 (2021).

29. Chi, X. et al. A neutralizing human antibody binds to the $\mathrm{N}$-terminal domain of the spike protein of SARS-CoV-2. Science 369, 650-655 (2020).

30. Greaney, A. J. et al. Comprehensive mapping of mutations to the SARS-CoV-2 receptor-binding domain that affect recognition by polyclonal human serum antibodies. Cell Host Microbe https://doi.org/10.1016/j.chom.2021.02.003 (2021).

31. Starr, T. N. et al. Deep mutational scanning of SARS-CoV-2 receptor binding domain reveals constraints on folding and ACE2 binding. Cell 182, 1295-1310 (2020).

32. Xie, X. et al. Neutralization of SARS-CoV-2 spike 69/70 deletion, E484K and N501Y variants by BNT162b2 vaccine-elicited sera. Nat. Med. https://doi.org/10.1038/s41591-02101270-4 (2021).

33. Rees-Spear, C. et al. The impact of spike mutations on SARS-CoV-2 neutralization. Preprint at https://doi.org/10.1101/2021.01.15.426849 (2021).

34. Wu, K. et al. mRNA-1273 vaccine induces neutralizing antibodies against spike mutants from global SARS-CoV-2 variants. Preprint at https://doi.org/10.1101/2021.01.25.427948 (2021).

35. Cele, S. et al. Escape of SARS-CoV-2 501Y.V2 variants from neutralization by convalescent plasma. Preprint at https://doi.org/10.1101/2021.01.26.21250224 (2021)

36. Wibmer, C. K. et al. SARS-CoV-2 501Y.V2 escapes neutralization by South African COVID-19 donor plasma. Nat. Med. https://doi.org/10.1038/s41591-021-01285-x (2021).

37. Wadman, M. \& Cohen, J. Novavax vaccine delivers $89 \%$ efficacy against COVID-19 in U.K.-but is less potent in South Africa. Science https://doi.org/10.1126/science.abg8101 (2021).

38. Callaway, E. \& Mallapaty, S. Novavax offers first evidence that COVID vaccines protect people against variants. Nature 590, 17 (2021)

39. Sabino, E. C. et al. Resurgence of COVID-19 in Manaus, Brazil, despite high seroprevalence. Lancet 397, 452-455 (2021)

40. Collier, D. et al. SARS-CoV-2 B.1.1.7 sensitivity to mRNA vaccine-elicited, convalescent and monoclonal antibodies. Preprint at https://doi.org/10.1101/2021.01.19.21249840 (2021).

41. Muik, A. et al. Neutralization of SARS-CoV-2 lineage B.1.1.7 pseudovirus by BNT162b2 vaccine-elicited human sera. Science https://doi.org/10.1126/science.abg6105 (2021)

42. Wang, Z. et al. mRNA vaccine-elicited antibodies to SARS-CoV-2 and circulating variants Nature https://doi.org/10.1038/s41586-021-03324-6 (2021).

43. Tada, T. et al. Neutralization of viruses with European, South African, and United States SARS-CoV-2 variant spike proteins by convalescent sera and BNT162b2 mRNA vaccine-elicited antibodies. Preprint at https://doi.org/10.1101/2021.02.05.430003 (2021).

44. Kemp, S. A. et al. SARS-CoV-2 evolution during treatment of chronic infection. Nature https://doi.org/10.1038/s41586-021-03291-y (2021).

45. McCarthy, K. R. et al. Recurrent deletions in the SARS-CoV-2 spike glycoprotein drive antibody escape. Science https://doi.org/10.1126/science.abf6950 (2021).

46. Choi, B. et al. Persistence and evolution of SARS-CoV-2 in an immunocompromised host. N. Engl. J. Med. 383, 2291-2293 (2020).

Publisher's note Springer Nature remains neutral with regard to jurisdictional claims in published maps and institutional affiliations.

(c) The Author(s), under exclusive licence to Springer Nature Limited 2021 


\section{Methods}

\section{Data reporting}

No statistical methods were used to predetermine sample size. The experiments were not randomized and the investigators were not blinded to allocation during experiments and outcome assessment.

\section{Patients and vaccines}

Plasma samples were obtained from patients (aged 34-79 years; mean, 54 years) convalescing from documented SARS-CoV-2 infection approximately one month after recovery or later. The patients were enrolled into an observational cohort study of patients convalescing from COVID-19 at the Columbia University Irving Medical Center (CUIMC) starting in the spring of 2020. The study protocol was approved by the CUIMC Institutional Review Board (IRB) and all participants provided written informed consent. From their documented clinical profiles, plasma samples from 10 patients with severe COVID-19 and plasma from 10 patients with a non-severe SARS-CoV-2 infection were selected for this study. Sera were obtained from 12 participants in a phase-I clinical trial of the Moderna SARS-CoV-2 mRNA-1273 vaccine conducted at the NIH, under a NIH IRB-approved protocol ${ }^{9}$. Sera were also obtained from 10 individuals followed in a CUIMC IRB-approved protocol to assess immunological responses to SARS-CoV-2 who received the Pfizer BNT162b2 COVID-19 vaccine as a part of the emergency use authorization.

\section{Monoclonal antibodies}

Monoclonal antibodies tested in this study were constructed and produced at Columbia University as previously described ${ }^{20}$, except REGN10933, REGN10987, REGN10985, COV2-2196 and COV2-2130, which were provided by Regeneron Pharmaceuticals, Brii-196 and Brii-198, which were provided by Brii Biosciences and CB6, which was provided by B.Z. and P.D.K.

\section{Authentic SARS-CoV-2 microplate neutralization}

The SARS-CoV-2 viruses USA-WA1/2020(WA1),USA/CA_CDC_5574/2020 (B.1.1.7) and hCoV-19/South Africa/KRISP-EC-K005321/2020 (B.1.351) were obtained from BEI Resources (NIAID, NIH) and propagated for one passage using Vero E6 cells. Virus infectious titres were determined by an end-point dilution and cytopathogenic effect assay on Vero E6 cells as previously described ${ }^{20}$.

An end-point dilution microplate neutralization assay was performed to measure the neutralization activity of convalescent plasma samples, sera from vaccinated individuals and purified monoclonal antibodies. In brief, plasma and serum samples were subjected to successive fivefold dilutions starting from 1:100. Similarly, most monoclonal antibodies were serially diluted (fivefold dilutions) starting at $10 \mu \mathrm{g} \mathrm{ml}^{-1}$. Some clinical antibodies were tested from starting concentrations of $1 \mu \mathrm{g} \mathrm{ml}^{-1}$. Triplicates of each dilution were incubated with SARS-CoV-2 at a multiplicity of infection of 0.1 in EMEM with $7.5 \%$ inactivated fetal calf serum for $1 \mathrm{~h}$ at $37^{\circ} \mathrm{C}$. After incubation, the virus-antibody mixture was transferred onto a monolayer of Vero E6 cells grown overnight. The cells were incubated with the mixture for around $70 \mathrm{~h}$. Cytopathogenic effects of viral infection were visually scored for each well in a blinded manner by two independent observers. The results were then converted into the percentage of neutralization at a given sample dilution or monoclonal antibody concentration, and the data (mean \pm s.e.m.) were plotted using a five-parameter dose-response curve in GraphPad Prism v.8.4.

\section{Construction and production of variant pseudoviruses}

The original pCMV3-SARS-CoV-2-spike plasmid was provided by P. Wang (Shandong University). Plasmids encoding the D614G variant, all of the single-mutation variants found in B.1.1.7 or B.1.351, the eightmutation-combination variant (B.1.1.7 $\Delta 8$ ) and nine-mutationcombination variant (B.1.351 $\Delta$ 9) were generated using the Quikchange II XL site-directed mutagenesis kit (Agilent). Recombinant Indiana VSV expressing different SARS-CoV-2 spike variants were generated as previously described ${ }^{20,21}$. HEK293T cells were grown to $80 \%$ confluency before transfection with the spike gene using Lipofectamine 3000 (Invitrogen). Cells were cultured overnight at $37^{\circ} \mathrm{C}$ with $5 \% \mathrm{CO}_{2}$ and VSV-G pseudo-typed $\Delta \mathrm{G}$-luciferase ( $\mathrm{G}^{*} \Delta \mathrm{G}$-luciferase, Kerafast) was used to infect the cells in DMEM at an multiplicity of infection of 3 for $2 \mathrm{~h}$ before washing the cells with $1 \times$ DPBS three times. The next day, the transfection supernatant was collected and clarified by centrifugation at $300 \mathrm{~g}$ for $10 \mathrm{~min}$. Each viral stock was then incubated with $20 \% \mathrm{I} 1$ hybridoma (anti-VSV-G; ATCC, CRL-2700) supernatant for $1 \mathrm{~h}$ at $37^{\circ} \mathrm{C}$ to neutralize contaminating the VSV-G pseudo-typed $\Delta \mathrm{G}$-luciferase virus before measuring titres and making aliquots to be stored at $-80^{\circ} \mathrm{C}$.

\section{Pseudovirus neutralization assays}

Neutralization assays were performed by incubating pseudoviruses with serial dilutions of monoclonal antibodies or heat-inactivated plasma or sera, and scored by the reduction in luciferase gene expression $^{20,21}$. In brief, Vero E6 cells were seeded in a 96-well plate at a concentration of $2 \times 10^{4}$ cells per well. Pseudoviruses were incubated the next day with serial dilutions of the test samples in triplicate for $30 \mathrm{~min}$ at $37^{\circ} \mathrm{C}$. The mixture was added to cultured cells and incubated for an additional $24 \mathrm{~h}$. The luminescence was measured by Luciferase Assay System (Promega). $\mathrm{IC}_{50}$ was defined as the dilution at which the relative light units were reduced by $50 \%$ compared with the virus control wells (virus + cells) after subtraction of the background in the control groups with cells only. The $\mathrm{IC}_{50}$ values were calculated using nonlinear regression in GraphPad Prism.

\section{Reporting summary}

Further information on research design is available in the Nature Research Reporting Summary linked to this paper.

\section{Data availability}

Materials used in this study will be made available but may require execution of a materials transfer agreement. All the data are provided in the paper or the Supplementary Information.

Acknowledgements We thank S. Goff and B. DeKosky for helpful discussions. This study was supported by funding from Andrew and Peggy Cherng, Samuel Yin, Barbara Picower and the JPB Foundation, Brii Biosciences, Roger and David Wu, and the Bill and Melinda Gates Foundation.

Author contributions The study was conceptualized by D.D.H. The experiments were mainly carried out by P.W., M.S.N., Y.H., L.L. and S.I. with assistance from M.W. and J.Y. Structural interpretations were made by Y.G., Z.S., L.S. and P.D.K. B.Z., P.D.K. and C.A.K. provided monoclonal antibodies. J.Y.C. and M.T.Y. provided plasma from patients convalescing from COVID-19. B.S.G. and J.R.M. provided sera from participants in the Moderna vaccine trial; J.Y.C. and M.S. provided sera from healthcare workers immunized with the Pfizer vaccine. Y.H. and Y.L. helped to supervise the study. The manuscript was written by D.D.H. with editing by P.W., P.D.K., L.S., Y.L. and was reviewed by, commented on and approved by all of the authors.

Competing interests P.W., L.L, J.Y., M.S.N., Y.H. and D.D.H. are inventors on a provisional patent application on monoclonal antibodies against SARS-CoV-2. D.D.H. is a member of the scientific advisory board of Brii Biosciences, which also has provided a grant to Columbia University to support this and other studies on SARS-CoV-2.

\section{Additional information}

Supplementary information The online version contains supplementary material available at https://doi.org/10.1038/s41586-021-03398-2.

Correspondence and requests for materials should be addressed to Y.H. or D.D.H. Peer review information Nature thanks Jesse D. Bloom, Andreas Radbruch and the other, anonymous, reviewer(s) for their contribution to the peer review of this work. Reprints and permissions information is available at http://www.nature.com/reprints. 
a

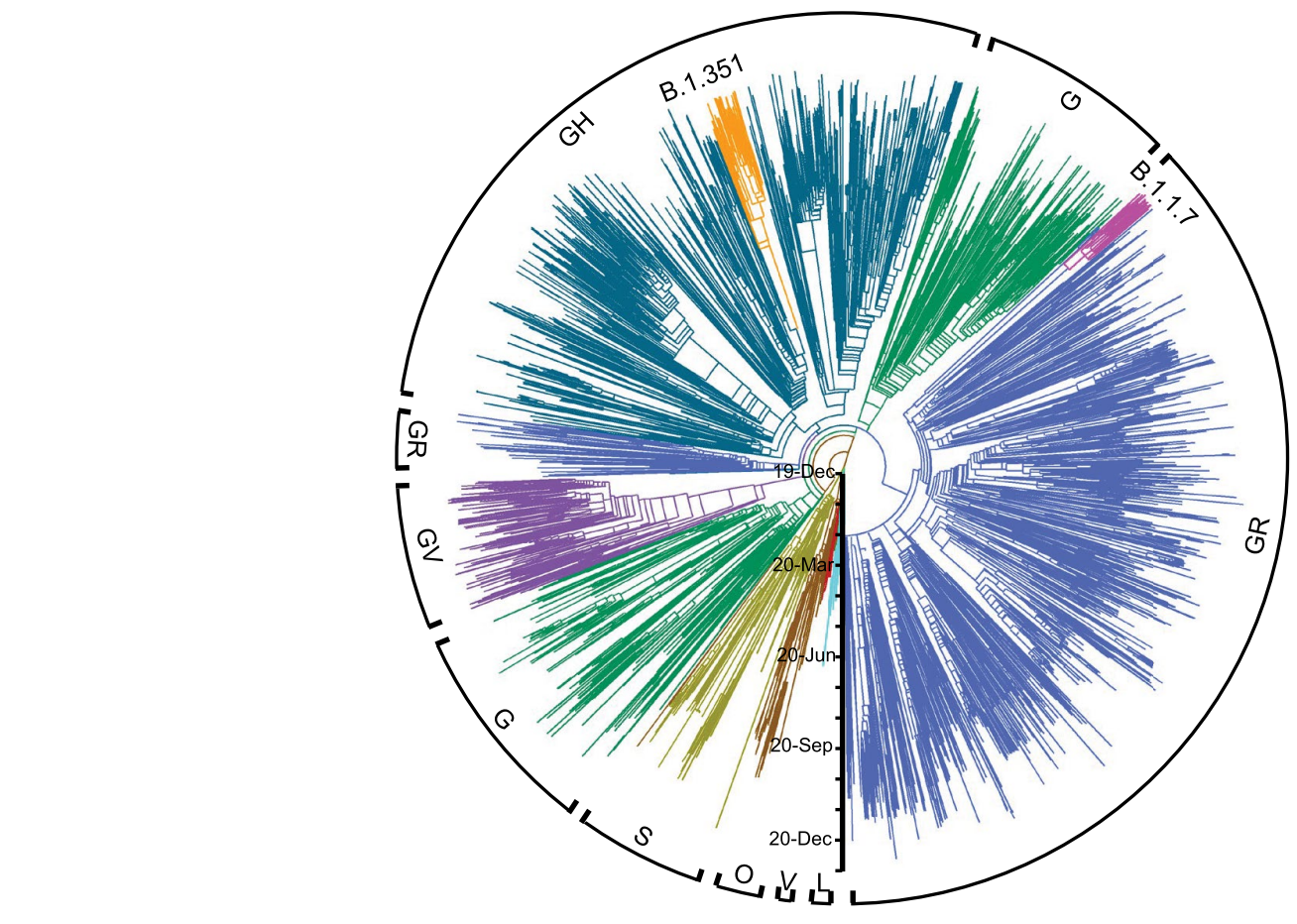

b

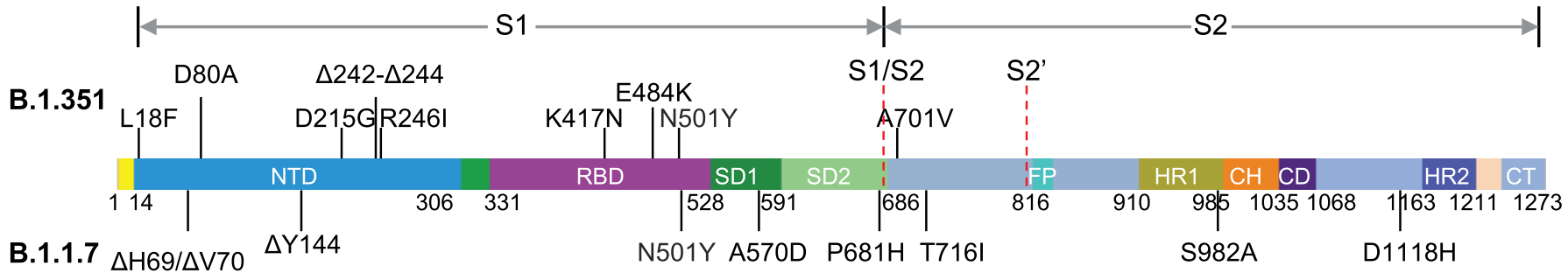

C

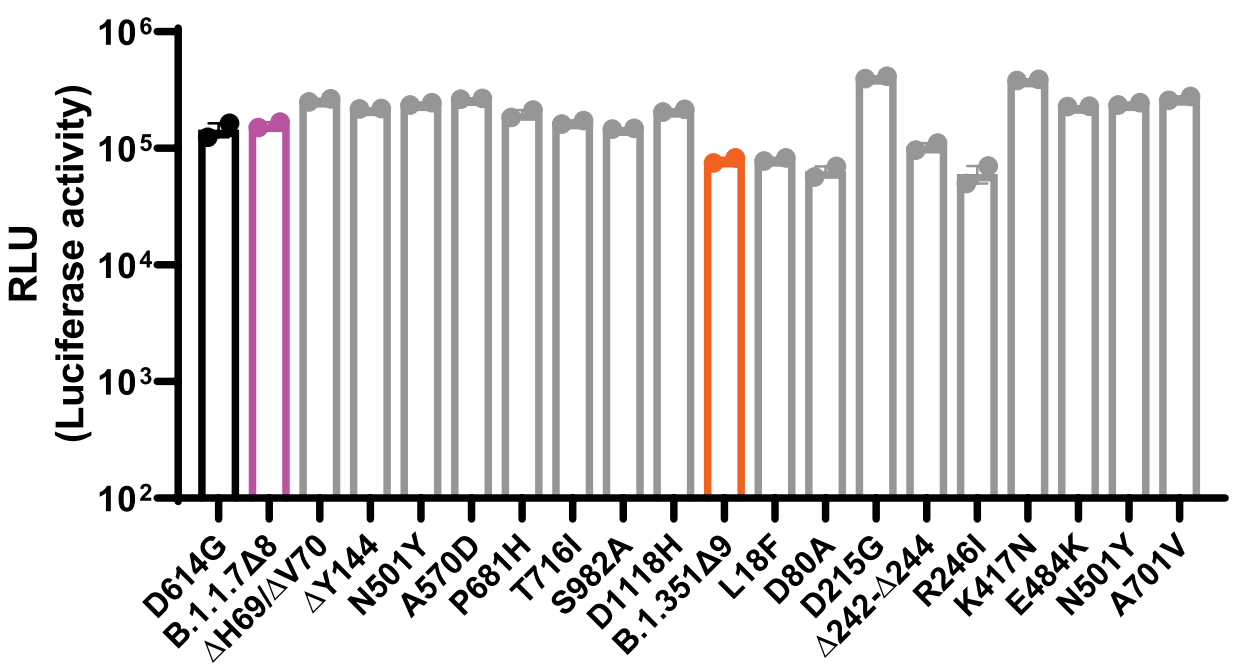

Extended Data Fig. 1 | Emerging SARS-CoV-2 variants identified in the UK and South Africa. a, Phylogenetic tree of SARS-CoV-2 variants, with B.1.351 and B.1.1.7 highlighted. $\mathbf{b}$, Mutations in the viral spike protein identified in B.1.351 (SA) and B.1.1.7 (UK) in addition to D614G.c, Titres of wild-type (D614G) and 18 mutant SARS-CoV-2 pseudoviruses. VSV-based pseudoviruses were generated ${ }^{20,21}$ and viral particles were quantified and normalized to the expression of the nucleocapsid protein of VSV by western blot. Equal amounts of each pseudovirus were then used to infect Vero E6 cells and relative luciferase units (RLUs) were measured $16 \mathrm{~h}$ later. Data are mean \pm s.e.m. of technical duplicates. 
a

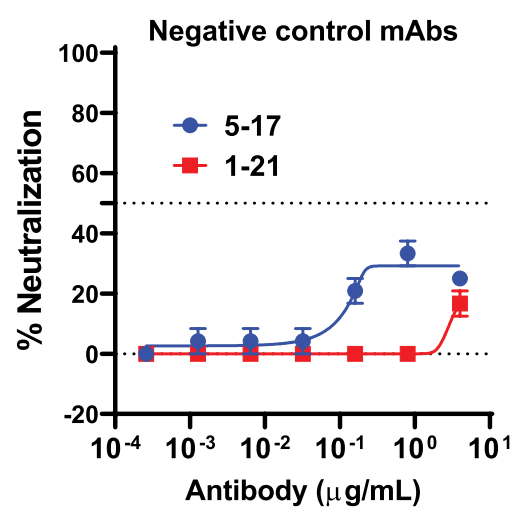

C

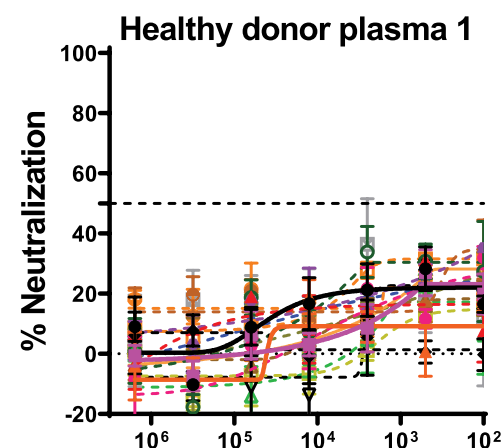

b

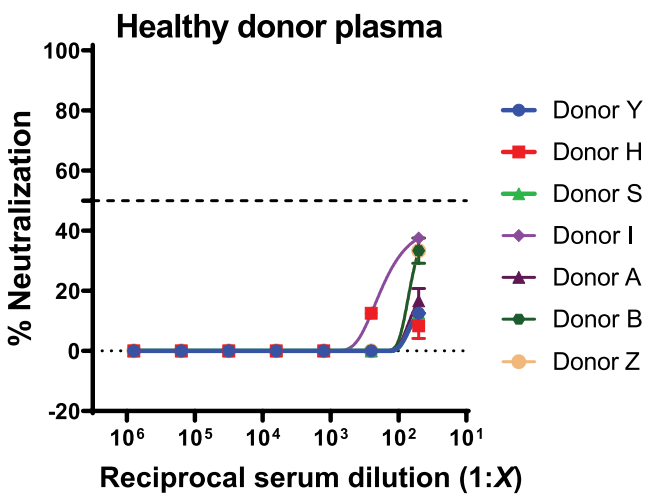

\section{Reciprocal plasma dilution (1:X)}

Extended Data Fig. 2 | Neutralization profiles of negative control monoclonal antibodies and plasma from healthy donors.

a,b, Neutralization of two SARS-CoV-2 non-neutralizing monoclonal antibodies ${ }^{20}$ (a) and healthy donor plasma against wild-type (WA1) live virus (b). c, Neutralization of plasma samples from two healthy donors against wild-type, B.1.1.7 8 and B.1.351 $\Delta 9$ viruses, as well as single-mutation pseudoviruses. Data are mean \pm s.e.m. of technical triplicates. 


\section{RBD mAbs}
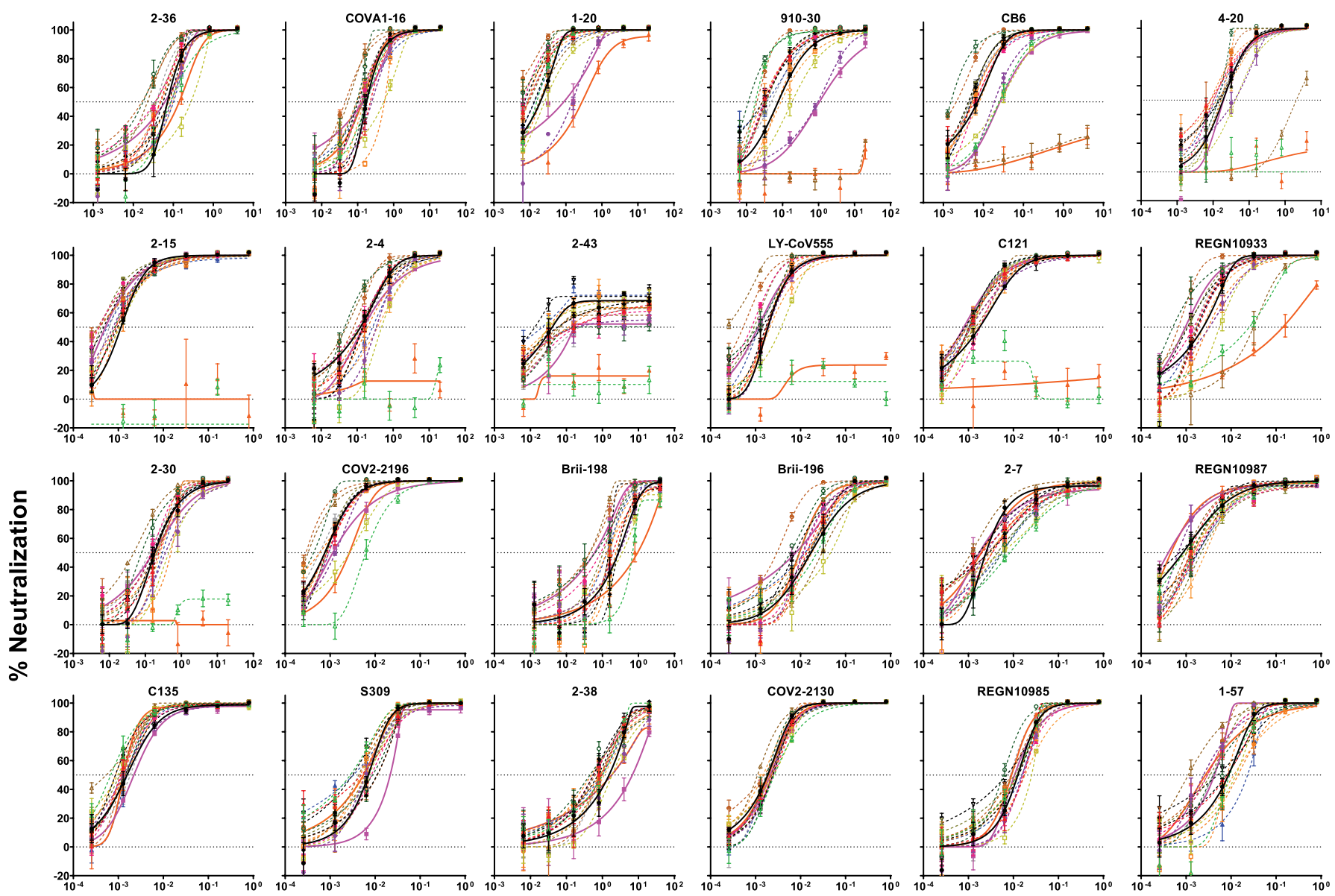

\section{NTD mAbs}
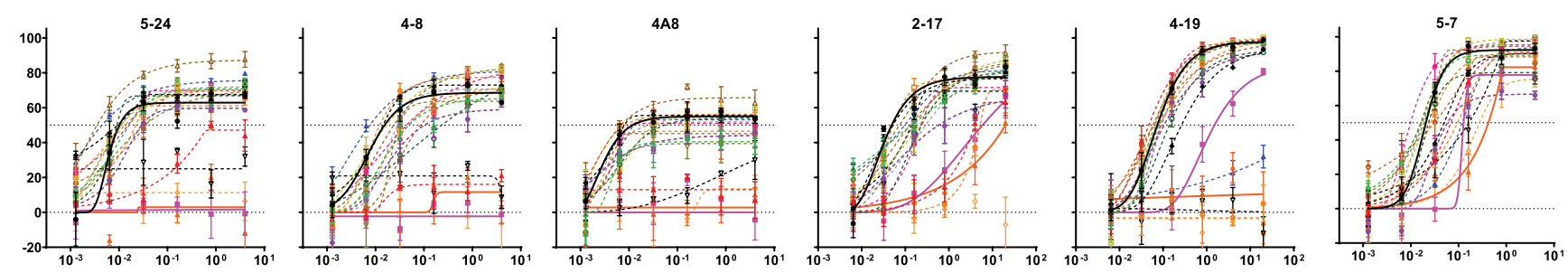

Antibody $(\mu \mathrm{g} / \mathrm{mL})$

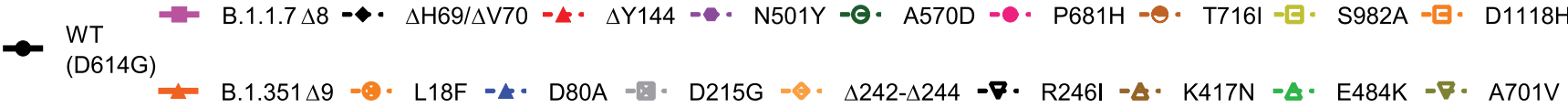

Extended Data Fig. 3 | Neutralization profiles of monoclonal antibodies against wild-type, B.1.1.7 8 and $B .1 .351 \Delta 9$ viruses, as well as single-mutation pseudoviruses. Data are mean \pm s.e.m. of technical triplicates. 


\section{Article}

a
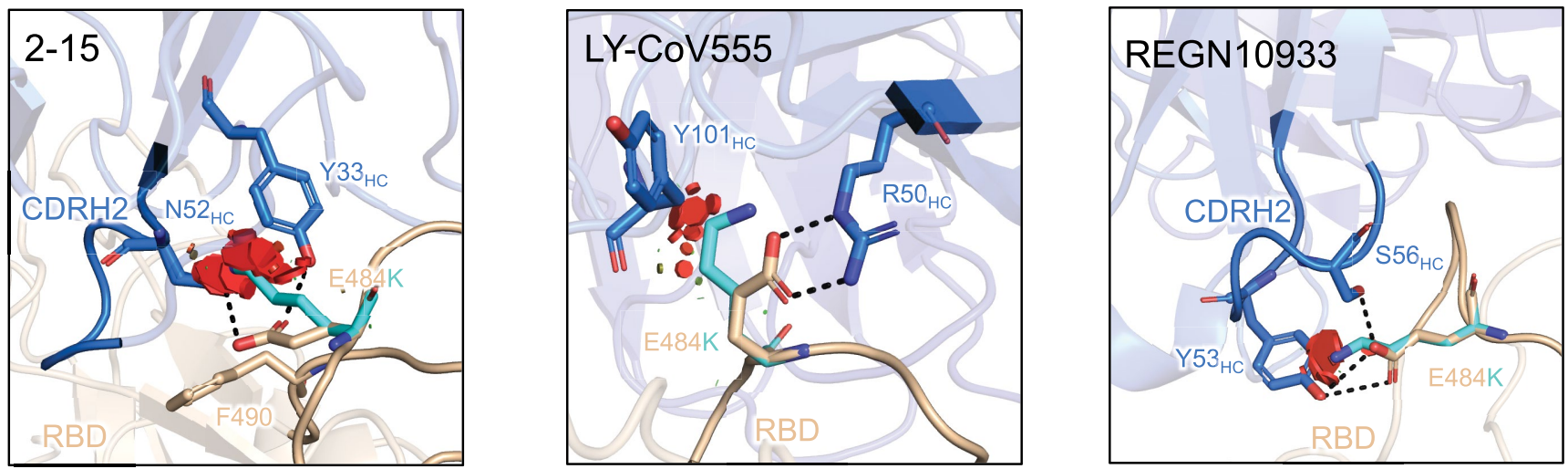

b

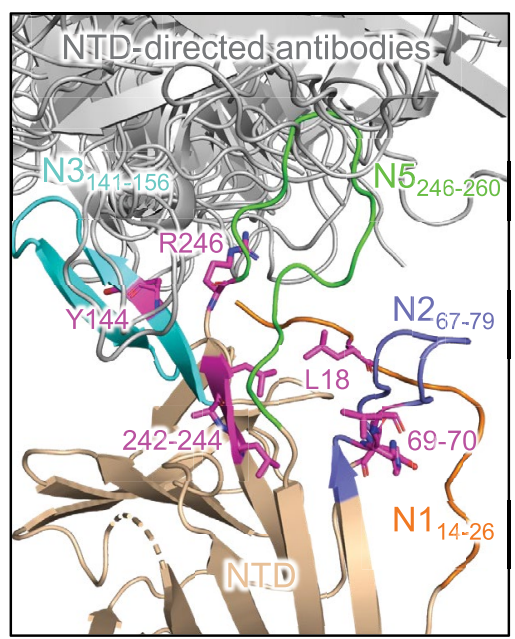

Extended Data Fig. 4 | Structural explanations on how critical mutations affect monoclonal antibody activity. a, E484 forms hydrogen bonds with monoclonal antibodies that target the RBM. E484K causes not only steric clashes but also a charge change at antibody-binding sites, and thus abolishes binding by these RBM-directed monoclonal antibodies. Steric clashes are shown by red plates. b. Mutations at or near the NTD antigenic supersitecomprising loops N1, N3 and N5-that is recognized by many potent NTD-directed monoclonal antibodies. 

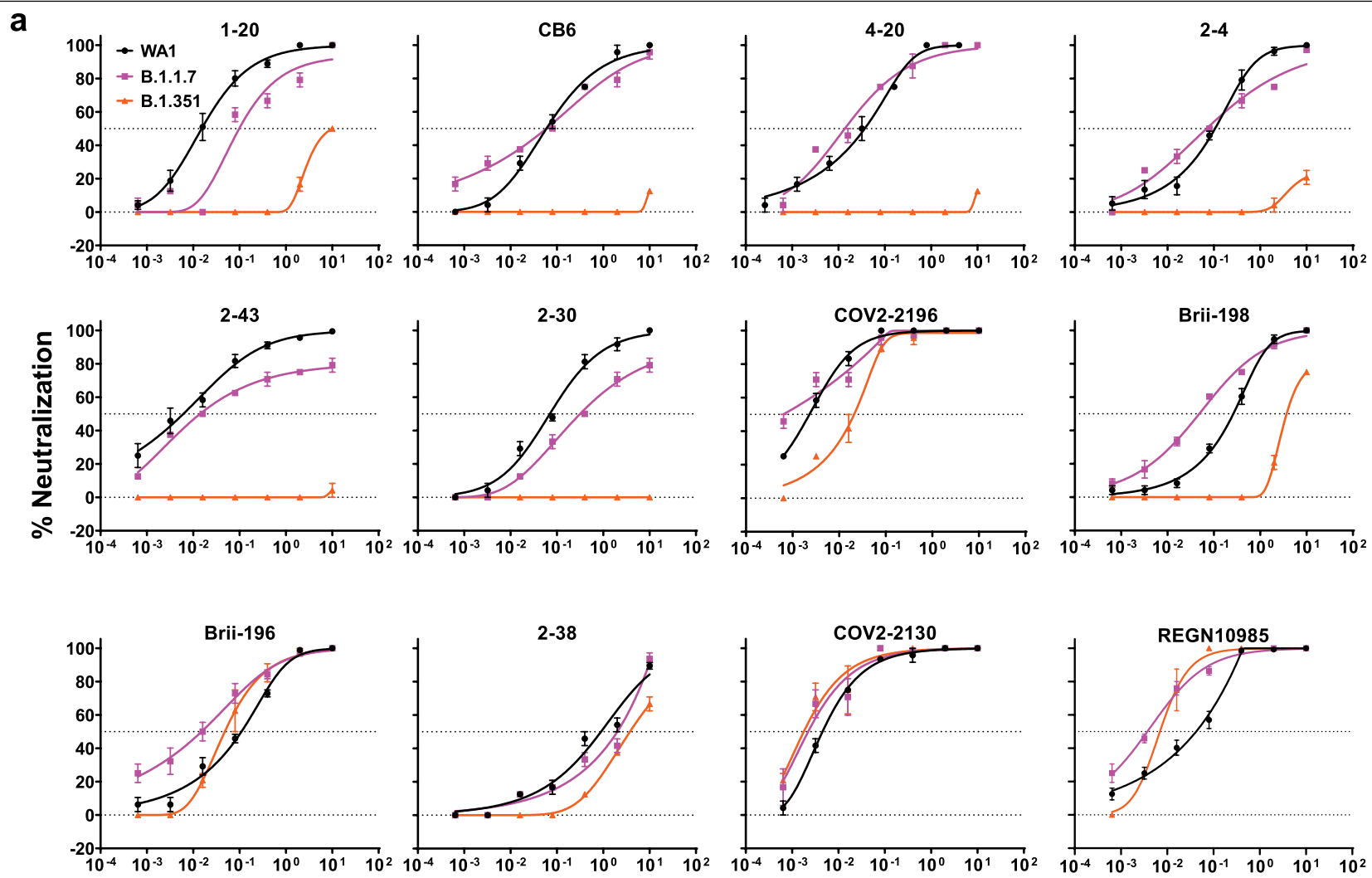

Antibody $(\mu \mathrm{g} / \mathrm{mL})$

b

\begin{tabular}{|c|c|c|c|c|c|c|c|c|c|c|c|c|}
\hline \multirow{2}{*}{$\begin{array}{l}\text { Fold change in } \mathrm{I}_{50} \\
\text { (compared to WT) }\end{array}$} & \multicolumn{12}{|c|}{ RBD-directed mAbs } \\
\hline & $1-20$ & CB6 & 4-20 & $2-4$ & $2-43$ & $2-30$ & cov2-2196 & Brii-198 & Brii-196 & $2-38$ & COV2-2130 & EEGN10985 \\
\hline B.1.1.7 & -5.6 & 1.4 & 2.4 & 1.3 & -2.3 & -4.4 & 3.0 & 5.6 & 7.3 & -1.8 & 3.0 & 10.5 \\
\hline 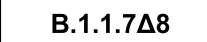 & -4.5 & -3.6 & -1.0 & -1.2 & -4.1 & 1.1 & -1.5 & 4.6 & 2.2 & -4.4 & -1.1 & 1.2 \\
\hline$\Delta \mathrm{H} 69 / \Delta \mathrm{V} 70$ & 1.5 & 1.5 & 1.2 & -1.2 & -1.4 & 1.1 & 1.0 & 1.1 & 1.1 & 2.8 & -1.3 & -1.0 \\
\hline$\Delta Y 144$ & 2.2 & 1.2 & 2.2 & -1.2 & -3.7 & -1.3 & -1.1 & 1.4 & 1.2 & 1.6 & -1.0 & -1.4 \\
\hline N501Y & -7.7 & -2.6 & -2.1 & -2.8 & -4.8 & -2.0 & -1.7 & -1.0 & 1.5 & -1.2 & -1.3 & -1.3 \\
\hline A570D & 4.6 & 4.6 & 1.4 & 2.7 & -5.3 & 2.3 & 1.8 & 4.4 & 2.9 & 3.4 & 1.1 & 2.1 \\
\hline $\mathrm{P} 681 \mathrm{H}$ & 2.9 & 1.1 & 1.8 & 1.2 & -4.5 & 1.3 & -1.2 & 3.1 & 1.5 & 2.7 & -1.4 & -1.3 \\
\hline T716I & 10.0 & 3.4 & 1.9 & 1.5 & -1.5 & -1.1 & 2.6 & 6.3 & 5.7 & 1.7 & 1.2 & 1.4 \\
\hline S982A & -1.3 & -3.6 & -2.2 & -3.9 & 1.2 & -2.4 & -3.6 & -1.3 & -2.7 & -1.2 & -1.4 & -2.0 \\
\hline $\mathrm{D} 1118 \mathrm{H}$ & 1.0 & 1.4 & 1.1 & -1.6 & 1.2 & -2.8 & 1.3 & 1.2 & 1.2 & 1.8 & -1.2 & 1.0 \\
\hline B 1 351 & -6670 & $<-1000$ & $<-1000$ & $<-1000$ & $<-1000$ & $<-1000$ & -63 & -146 & 22 & 25 & 30 & 53 \\
\hline B.1.351 $\Delta 9$ & -14.4 & $<-1000$ & $<-1000$ & $<-1000$ & $<-1000$ & $<-1000$ & -3.5 & -3.4 & 1.8 & -1.2 & -1.0 & 1.4 \\
\hline L18F & 2.2 & 1.3 & 2.2 & -1.0 & -2.8 & -1.1 & 1.0 & 2.1 & 1.1 & 3.1 & -1.2 & 1.1 \\
\hline D80A & 1.4 & 1.4 & 1.6 & -2.4 & 1.9 & -1.1 & -1.2 & 2.1 & 1.4 & 1.6 & -1.0 & -1.2 \\
\hline D215G & 2.4 & 1.6 & 1.3 & -1.4 & -5.6 & 1.4 & 1.3 & 1.7 & 1.0 & 1.5 & 1.1 & 1.4 \\
\hline$\Delta 242-\Delta 244$ & 5.1 & -1.1 & 1.5 & -2.1 & 1.2 & 1.1 & 1.0 & 1.7 & -1.1 & 1.4 & 1.3 & -1.4 \\
\hline R246I & 2.8 & 1.7 & 1.3 & -1.1 & 3.0 & 1.2 & -1.1 & 1.9 & 2.3 & 2.0 & -1.2 & 1.8 \\
\hline K417N & 1.1 & $<-1000$ & -108.3 & 2.5 & -1.7 & 3.8 & 2.4 & 6.3 & -1.8 & 2.5 & 1.7 & 1.1 \\
\hline E484K & 1.3 & -3.5 & $<-1000$ & $<-1000$ & $<-1000$ & $<-1000$ & -8.1 & -2.5 & -1.4 & 1.5 & -1.4 & 1.0 \\
\hline N501Y & -7.7 & -2.6 & -2.1 & -2.8 & -4.8 & -2.0 & -1.7 & -1.0 & 1.5 & -1.2 & -1.3 & -1.3 \\
\hline A701V & 2.1 & 1.6 & 1.1 & 1.1 & -1.6 & -1.2 & -1.1 & 2.7 & 1.8 & 1.7 & -1.0 & 1.2 \\
\hline
\end{tabular}

Extended Data Fig. 5 | Neutralization susceptibility of B.1.1.7 and B.1.351 variants to additional SARS-CoV-2 RBD-directed monoclonal antibodies. a, Neutralization of B.1.1.7, B.1.351 and wild-type viruses by additional RBD-directed monoclonal antibodies. Data are mean \pm s.e.m. of technical triplicates. b. Fold increase or decrease in $\mathrm{IC}_{50}$ of neutralizing monoclonal antibodies against B.1.1.7 and B.1.351, as well as mutant pseudoviruses, relative to the wild type. Red, resistance $>3$-fold; green, sensitization $>3$-fold. 
a

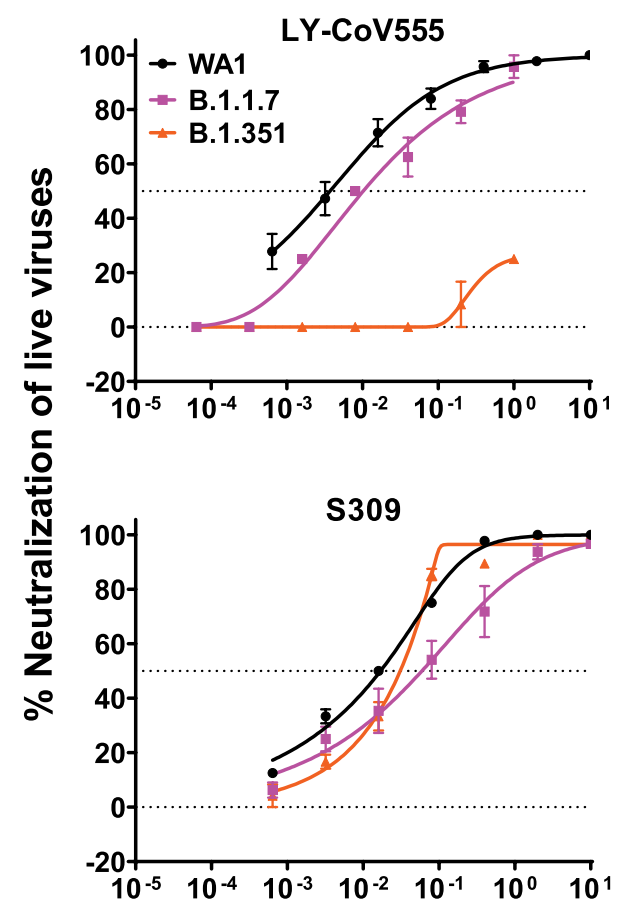

b

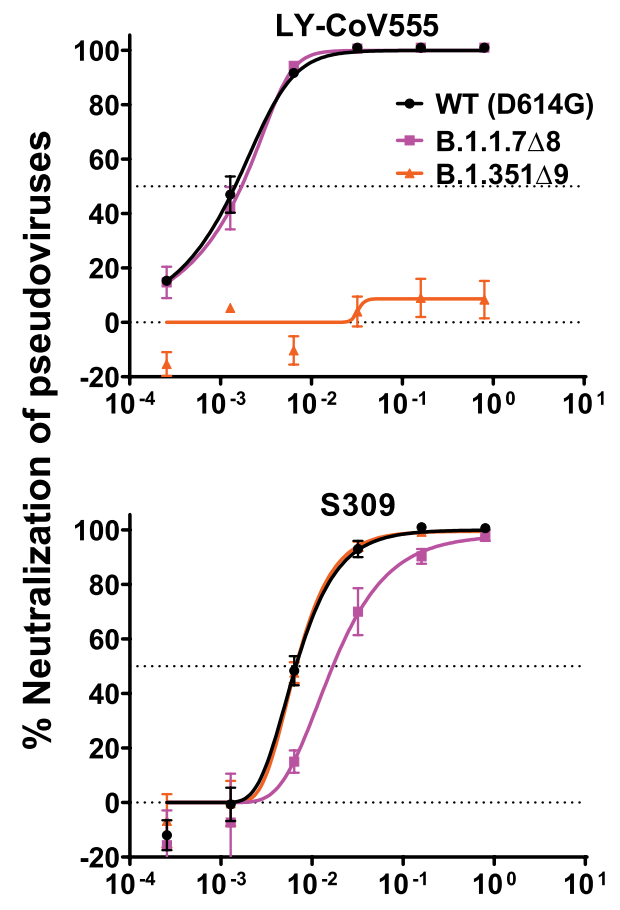

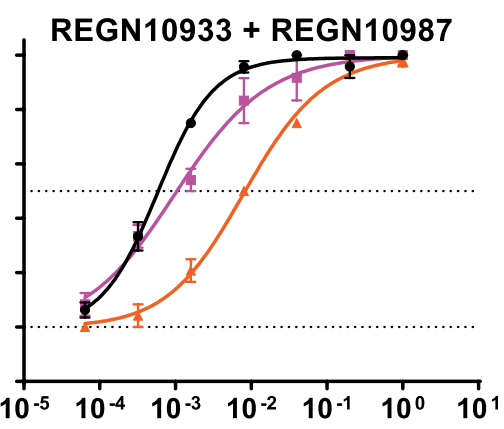
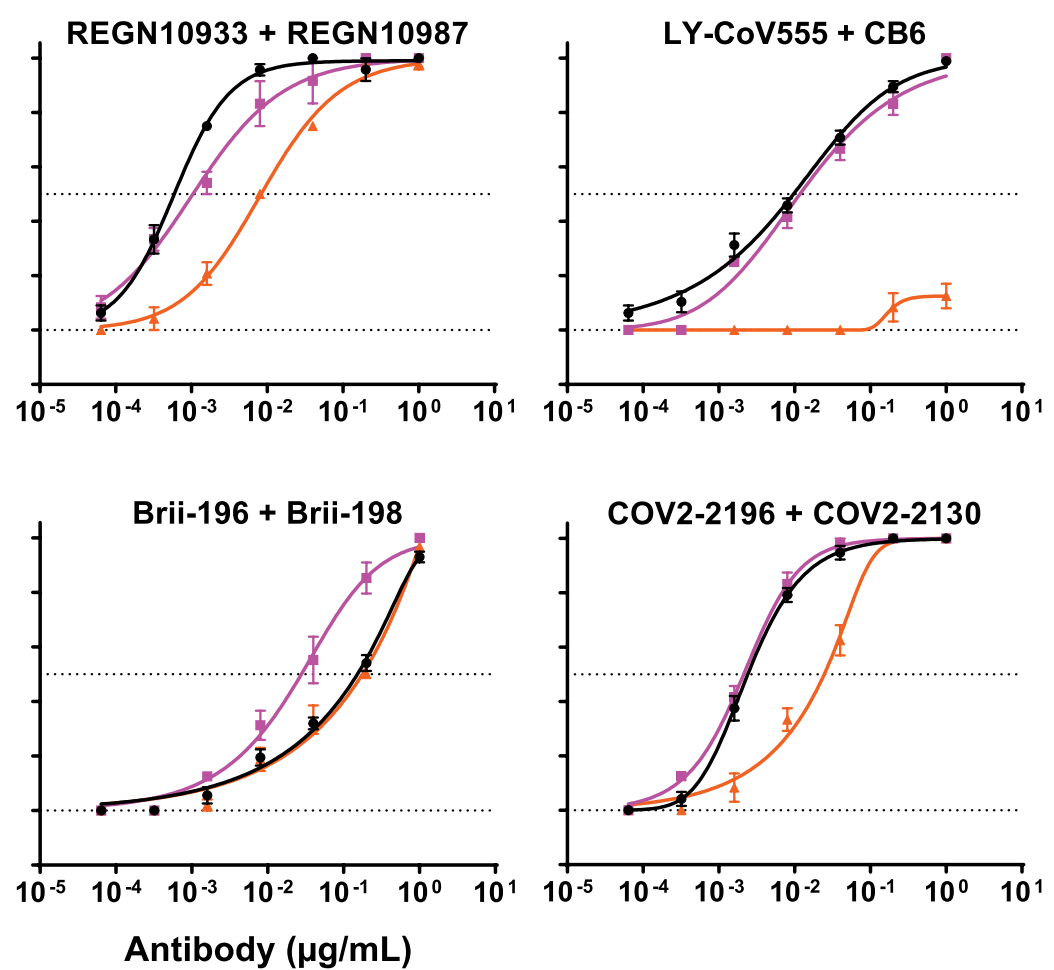

Antibody ( $\mu \mathrm{g} / \mathrm{mL})$
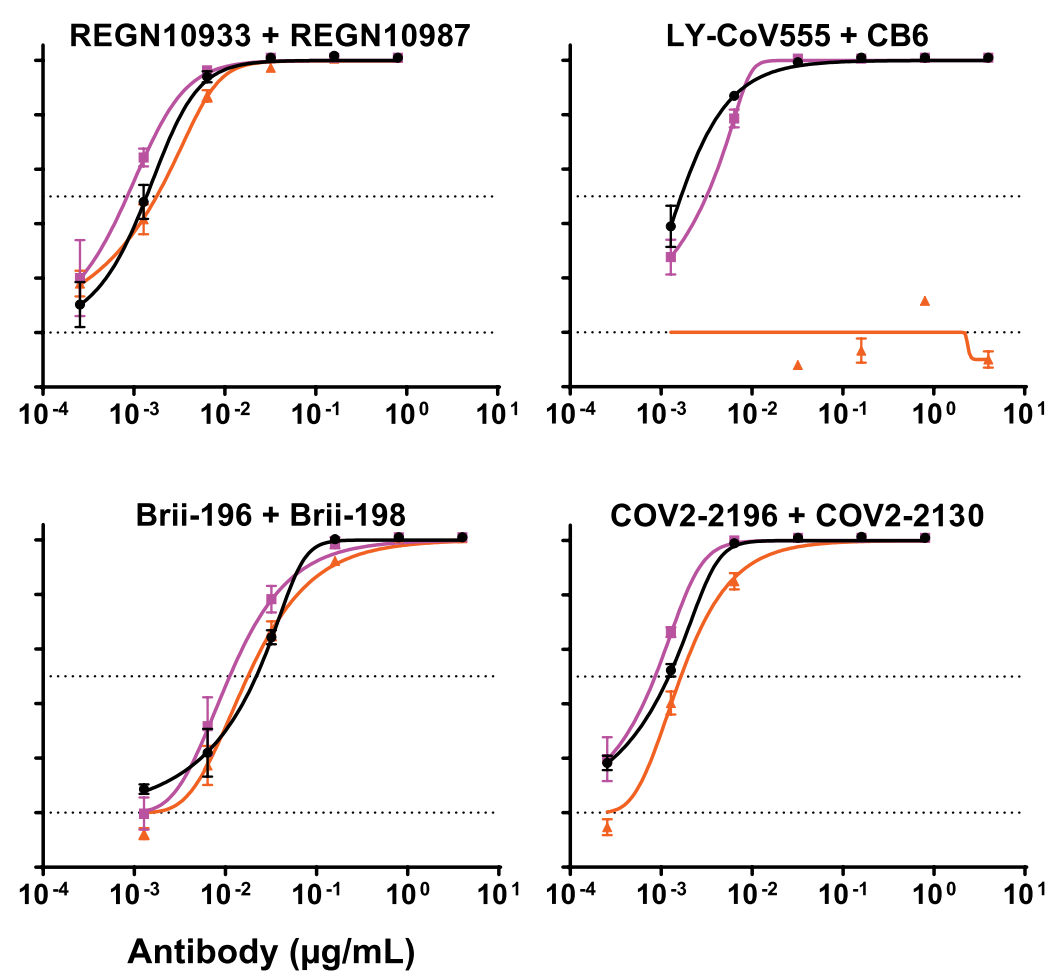

Antibody $(\mu \mathrm{g} / \mathrm{mL})$

Extended Data Fig. 6 | Neutralization profiles of authorized or investigational therapeutic monoclonal antibodies against live viruses and pseudoviruses. a, Wild-type (WA1), B.1.1.7, and B.1.351 live viruses.b, Wild-type (D614G), B.1.1.7 8 and B.1.351 $\Delta 9$ pseudoviruses. Data are mean \pm s.e.m. of technical triplicates. 


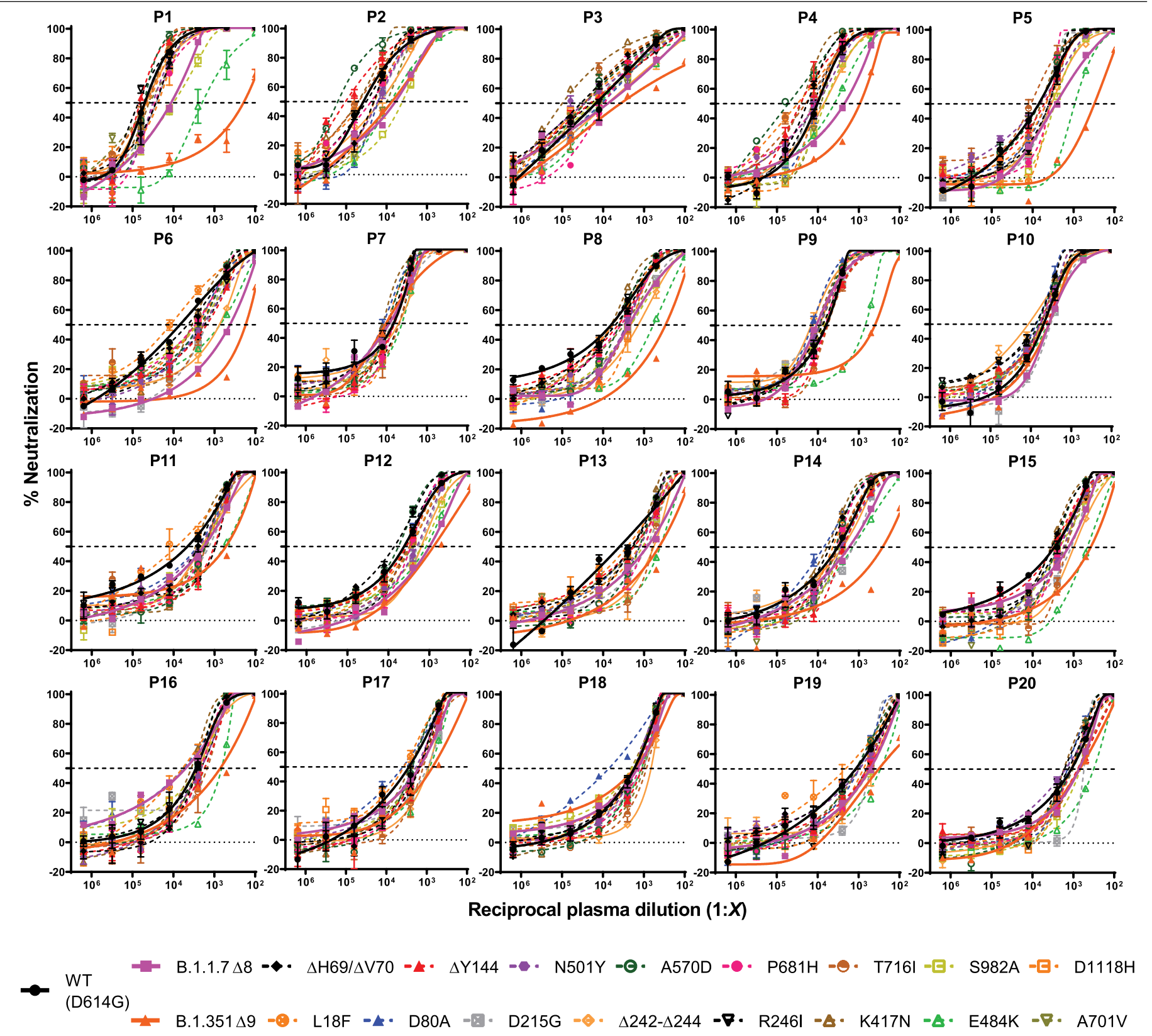

Extended Data Fig. 7 | Neutralization profiles of plasma samples of 20 patients convalescing from COVID-19 against wild-type, B.1.1.7 $\Delta 8$,

B.1.351 $\Delta 9$ and single-mutation pseudoviruses. Data are mean \pm s.e.m. of technical triplicates. Neutralization by plasma from healths donor is shown in Extended Data Fig. 2c. 

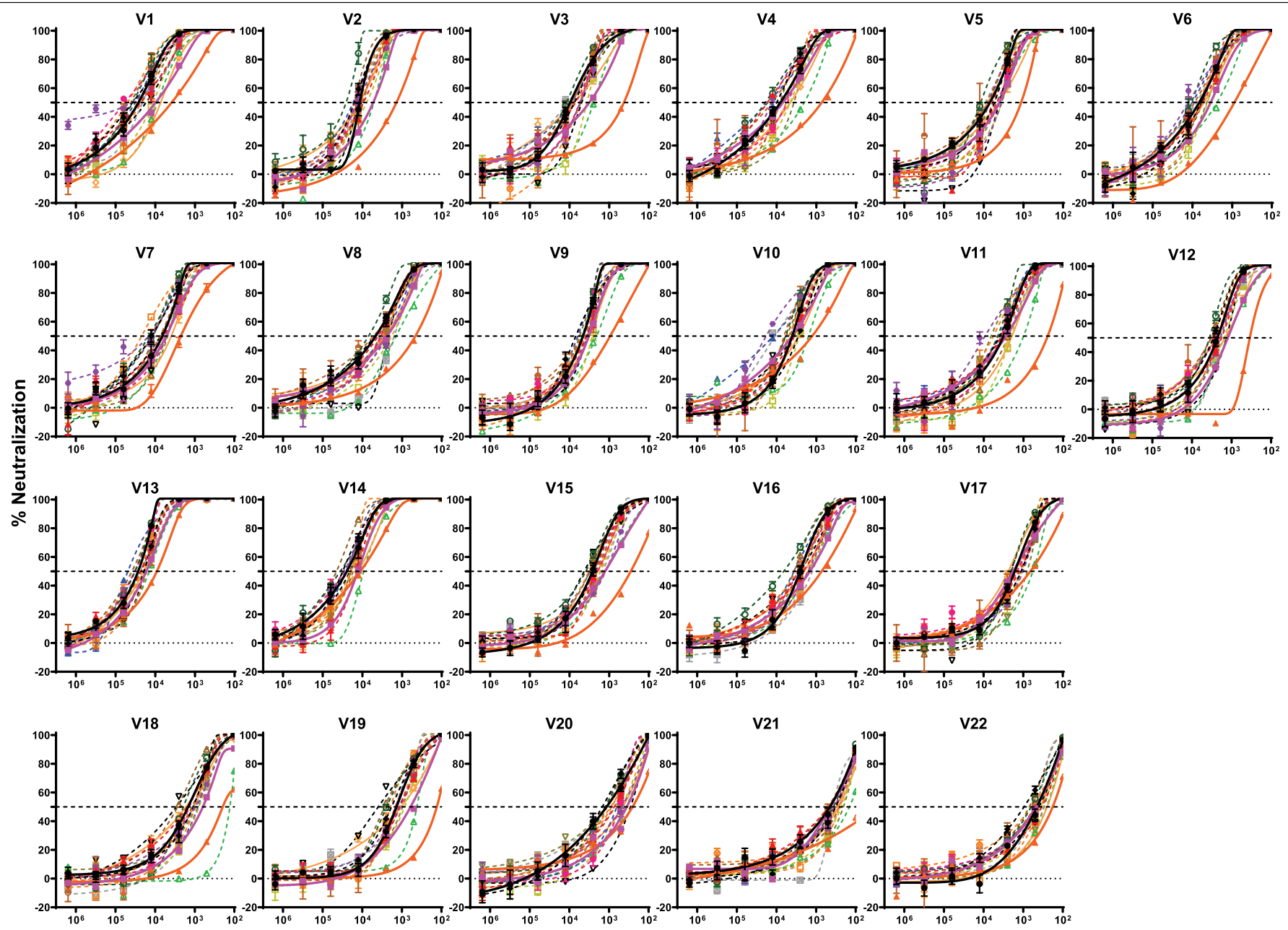

Reciprocal serum dilution (1:X)

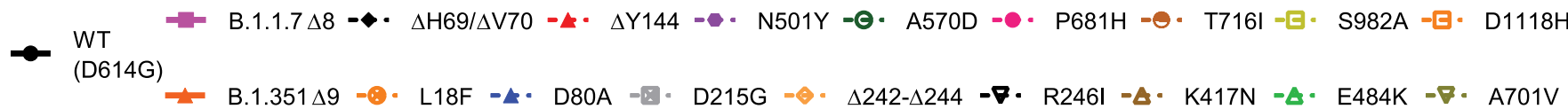

Extended Data Fig. 8 | Neutralization profiles of sera from vaccinated

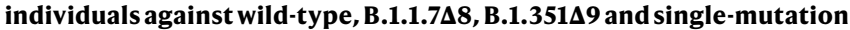
pseudoviruses. We tested 12 sera from individuals who received the Moderna vaccine (V1-V12) and 10 sera from individuals who received the Pfizer vaccine (V13-V22). Data are mean \pm s.e.m. of technical triplicates. 


\section{Reporting Summary}

Nature Research wishes to improve the reproducibility of the work that we publish. This form provides structure for consistency and transparency in reporting. For further information on Nature Research policies, see our Editorial Policies and the Editorial Policy Checklist.

\section{Statistics}

For all statistical analyses, confirm that the following items are present in the figure legend, table legend, main text, or Methods section.

n/a Confirmed

$\square$ The exact sample size $(n)$ for each experimental group/condition, given as a discrete number and unit of measurement

$\square$ A statement on whether measurements were taken from distinct samples or whether the same sample was measured repeatedly

$\square$ The statistical test(s) used AND whether they are one- or two-sided

Only common tests should be described solely by name; describe more complex techniques in the Methods section.

$\bigotimes \square$ A description of all covariates tested

$\bigotimes \square$ A description of any assumptions or corrections, such as tests of normality and adjustment for multiple comparisons

$\triangle$ A full description of the statistical parameters including central tendency (e.g. means) or other basic estimates (e.g. regression coefficient)

AND variation (e.g. standard deviation) or associated estimates of uncertainty (e.g. confidence intervals)

$\square$ For null hypothesis testing, the test statistic (e.g. $F, t, r$ ) with confidence intervals, effect sizes, degrees of freedom and $P$ value noted

Give $P$ values as exact values whenever suitable.

$\bigotimes \square$ For Bayesian analysis, information on the choice of priors and Markov chain Monte Carlo settings

$\bigotimes \square$ For hierarchical and complex designs, identification of the appropriate level for tests and full reporting of outcomes

$\bigotimes \square$ Estimates of effect sizes (e.g. Cohen's $d$, Pearson's $r$ ), indicating how they were calculated

Our web collection on statistics for biologists contains articles on many of the points above.

\section{Software and code}

Policy information about availability of computer code

Data collection SoftMax Pro 7.0.2 (Molecular Devices, LL) was used to measure luminescence in the pseudovirus neutralization assays

Data analysis This study used commercially available GraphPad Prism software v8.4 for data representation and statistical analysis (GraphPad Prism; RRID: SCR_002798).

For manuscripts utilizing custom algorithms or software that are central to the research but not yet described in published literature, software must be made available to editors and reviewers. We strongly encourage code deposition in a community repository (e.g. GitHub). See the Nature Research guidelines for submitting code \& software for further information.

\section{Data}

Policy information about availability of data

All manuscripts must include a data availability statement. This statement should provide the following information, where applicable:

- Accession codes, unique identifiers, or web links for publicly available datasets

- A list of figures that have associated raw data

- A description of any restrictions on data availability

Materials used in this study will be made available but may require execution of a materials transfer agreement. Source data are provided herein. 


\section{Field-specific reporting}

Please select the one below that is the best fit for your research. If you are not sure, read the appropriate sections before making your selection. $\bigotimes$ Life sciences $\quad \square$ Behavioural \& social sciences $\quad \square$ Ecological, evolutionary \& environmental sciences

For a reference copy of the document with all sections, see nature.com/documents/nr-reporting-summary-flat.pdf

\section{Life sciences study design}

All studies must disclose on these points even when the disclosure is negative.

Sample size

We obtained convalescent plasma from 20 patients, vaccinee sera from 12 Moderna SARS-Co-2 mRNA-1273 Vaccine participants and 10 Pfizer BNT162b2 Covid-19 Vaccine participants. The sample size is appropriate within technical capability to compare the difference between groups

Data exclusions No data were excluded from the analysis

Replication Studies that were repeated are noted in figure captions and include all studies that demonstrated the key results reported in the manuscript.

Randomization Randomization is not relevant as this is an observational study.

Blinding The investigators were not blinded as this is an observational study.

\section{Reporting for specific materials, systems and methods}

We require information from authors about some types of materials, experimental systems and methods used in many studies. Here, indicate whether each material, system or method listed is relevant to your study. If you are not sure if a list item applies to your research, read the appropriate section before selecting a response.

Materials \& experimental systems

\begin{tabular}{l|l}
\hline Involved in the study \\
\hline
\end{tabular}

\begin{tabular}{l|l}
\multicolumn{2}{l}{ Methods } \\
\hline n/a & Involved in the study \\
$\searrow$ & $\square$ ChIP-seq \\
$\searrow$ & $\square$ Flow cytometry \\
$\square$ & $\square$ MRI-based neuroimaging
\end{tabular}

\section{Antibodies}

Antibodies used

Monoclonal antibodies tested in this study were constructed and produced at Columbia University as previously described20, except REGN10933, REGN10987, REGN10985, COV2-2196, and COV2-2130 were provided by Regeneron Pharmaceuticals, Inc., Brii-196 and Brii-198 were provided by Brii Biosciences, and CB6 was provided by B.Z. and P.D.K. Most mAbs were serially diluted (5-fold dilutions) starting at $10 \mu \mathrm{g} / \mathrm{mL}$. Some clinical antibodies were tested from starting concentrations of $1 \mu \mathrm{g} / \mathrm{mL}$.

Validation

All of the SARS-CoV2 spike antigen-specific monoclonal antibodies have been validated by binding to SARS-CoV-2 spike and neutralizing SARS-CoV-2 pseudovirus in previous publications cited in this paper.

\section{Eukaryotic cell lines}

Policy information about cell lines

Cell line source(s)

In this study we used the following cell lines: Vero E6 (ATCC, Cat\# CRL-1586) and HEK293T (ATCC Cat\# CRL-3216).

Authentication

Mycoplasma contamination

Commonly misidentified lines (See ICLAC register)
All cell lines were obtained from authenticated vendors. Cells were recovered as healthy logarithmically growing cells within 4 to 7 days after thawing. Viability was measured and found to be $>90 \%$.

Mycoplasma is negative (Detected mycoplasma contamination using Mycoplasma PCR ELISA, Sigma,catalog number is 11663925910)

None 
Policy information about studies involving human research participants

Population characteristics

Plasma samples were obtained from patients (age 34-79; mean 54) convalescing from documented SARS-CoV-2 infection approximately one month after recovery or later.

Recruitment

Convalescing patients volunteered for the cohort study. These cases were enrolled into an observational cohort study of convalescent patients followed at the Columbia University Irving Medical Center starting in the Spring of 2020. From their documented clinical profiles, plasma samples from ten with severe Covid-19 were selected, along with plasma from 10 with non-severe infection, for this study.

Ethics oversight

The study protocol was approved by the CUIMC Institutional Review Board (IRB), and all participants provided written informed consent.

Sera were obtained from 12 participants in a Phase 1 clinical trial of Moderna SARS-CoV-2 mRNA-1273 Vaccine conducted at the $\mathrm{NIH}$, under a NIH IRB-approved protocol. Sera were also obtained from 10 individuals followed in a CUIMC IRB-approved protocol to assess immunological responses to SARS-CoV-2 who received the Pfizer BNT162b2 Covid-19 Vaccine as a part of the emergency use authorization.

Note that full information on the approval of the study protocol must also be provided in the manuscript. 